\title{
The Concentric Maclaurin Spheroid method with tides and a rotational enhancement of Saturn's tidal response
}

\author{
Sean M. Wahla, ${ }^{\mathrm{a},}$, William B. Hubbard ${ }^{\mathrm{b}}$, Burkhard Militzer ${ }^{\mathrm{a}, \mathrm{c}}$ \\ ${ }^{a}$ Department of Earth and Planetary Science, The University of California, Berkeley, CA, \\ 94720-4767, USA \\ ${ }^{b}$ Lunar and Planetary Laboratory, The University of Arizona, Tucson, AZ 85721-0092, \\ $U S A$ \\ ${ }^{c}$ Department of Astronomy, The University of California, Berkeley, CA, 94720-4767, USA
}

\begin{abstract}
We extend to three dimensions the Concentric Maclaurin Spheroid method for obtaining the self-consistent shape and gravitational field of a rotating liquid planet, to include a tidal potential from a satellite. We exhibit, for the first time, an important effect of the planetary rotation rate on tidal response of gas giants, whose shape is dominated by the centrifugal potential from rapid rotation. Simulations of planets with fast rotation rates like those of Jupiter and Saturn, exhibit significant changes in calculated tidal love numbers $k_{n m}$ when compared with non-rotating bodies. A test model of Saturn fitted to observed zonal gravitational multipole harmonics yields $k_{2}=0.413$, consistent with a recent observational determination from Cassini astrometry data (Lainey et al. 2016). The calculated love number is robust under reasonable assumptions of interior rotation rate, satellite parameters, and details of Saturn's interior structure. The method is benchmarked against several published test cases.
\end{abstract}

Keywords: Jovian planets, Tides, Interiors, Saturn

\section{Introduction}

The gas giants Jupiter and Saturn rotate so rapidly that adequate treatment of the non-spherical part of their gravitational potential requires either a

${ }^{*}$ Corresponding author
Email address: swahl@berkeley.edu (Sean M. Wahl )

Preprint submitted to Icarus

May 20, 2016

(C) 2016. This manuscript version is made available under the Elsevier user license http://www.elsevier.com/open-access/userlicense/1.0/ 
very high-order perturbative, or better, an entirely non-perturbative approach

5 (Hubbard, 2012, 2013, Hubbard et al., 2014, Wisdom, 1996, Wisdom and Hubbard, 2016). Here we present an extension of the Concentric Maclaurin Spheroid (CMS) method of Hubbard (2012, 2013) to three dimensions to include the tidal perturbation from a satellite. This allows for high-precision simulations of static tidal response, consistent with the planet's shape and interior mass distribution.

10 The presence of a large rotational bulge produces an observable effect on the tidal response of giant planets. This effect, which has not been previously revealed by linear tidal-response theories applied to spherical-equivalent interior models, has implications for the observed tidal responses of Jupiter and Saturn.

The Juno spacecraft is expected to measure the strength of Jupiter's gravitational field to an unprecedented precision $\left(\sim\right.$ one part in $\left.10^{9}\right)$ (Kaspi et al. 2010), potentially revealing a weak signal from the planet's interior dynamics. Also present in Jupiter's gravitational field will be tesseral-harmonic terms produced by tides raised by the planet's large satellites. In fact, close to the planet, the gravitational signal from Jupiter's tides has a similar magnitude to the predicted signal from models of deep internal dynamics Cao and Stevenson, 2015, Kaspi et al. 2010, Kaspi, 2013). An accurate prediction of the planet's hydrostatic tidal response will, therefore, be essential for interpreting the highprecision measurements provided by the Juno gravity science experiment.

Although the Cassini Saturn orbiter was not designed for direct measurement of high-order components of Saturn's gravitational field, it has already provided gravitational information relevant to the planet's interior structure. Lainey et al. (2016) used an astrometry dataset of the orbits of Saturn's coorbital satellites to make the first determination of the planet's $k_{2}$ love number. Their observed $k_{2}$ was significantly larger than the theoretical prediction of 30 Gavrilov and Zharkov (1977). A mismatch between an observed $k_{2}$ and the value predicted for a Saturn model fitted to the planet's low-degree zonal harmonics $J_{2}$ and $J_{4}$ would raise questions about the adequacy of the hydrostatic (non-dynamic) theory of tides.

In this paper we present theoretical results for simplified Saturn interior 
models matching the planet's observed low-degree zonal harmonics. When these models are analyzed with the full 3-d CMS theory including rotation and tides, we predict a gravitational response in line with the observed $k_{2}$ value of Lainey et al. (2016), suggesting that the observation can be completely understood in terms of a static tidal response. A similar test will be possible for Jupiter once 40 its $k_{2}$ has been measured by the Juno spacecraft.

There is extensive literature on the problem of the shape and gravitational potential of a liquid planet in hydrostatic equilibrium, responding to its own rotation and to an external gravitational potential from a satellite; see, e.g., a century-old discussion in Jeans (2009). Many classical geophysical investigations

45 use a perturbation approach, obtaining the planet's linear and higher-order response to small deviations of the potential from spherical symmetry. A good discussion of the application of perturbation theory to rotational response, the so-called theory of figures, is found in Zharkov and Trubitsyn (1978), while a pioneering calculation of the tidal response of giant planets is presented by 50 Gavrilov and Zharkov (1977).

Hubbard (2012) introduced an iterative numerical method, based on the theory of figures, for calculating the self-consistent shape and gravitational field of a constant density, rotating fluid body to high precision. In the CMS method, integrals over the mass distribution are solved using Gaussian quadrature to obtain the gravitational multipole moments. This method was extended to nonconstant density profiles by Hubbard (2013), by approximating the barotropic pressure-density relationship with multiple concentric constant-density (Maclaurin) spheroids. Here a spheroid is defined as a smooth shape obtained from deforming a sphere in three dimensions and is more general than an ellipsoid, whose shape is uniquely defined by 3 parameters. This approach mitigates problems with cancellation of terms that arise in a purely numerical solution to the general equation of hydrostatic equilibrium, and has a typical relative precision of $\sim 10^{-12}$. The CMS method has been benchmarked against analytical results for simple models (Hubbard et al. 2014) and against an independent, non65 perturbative numerical method (Wisdom, 1996; Wisdom and Hubbard, 2016). 
The theory of Gavrilov and Zharkov (1977) begins with an interior model of Saturn fitted to the values of $J_{2}$ and $J_{4}$ observed at that time. This interior model tabulates the mass density $\rho$ as a function of $s$, where $s$ is the mean radius of the constant-density surface. Tidal perturbation theory is then applied to this spherical-equivalent Saturn. The Gavrilov and Zharkov (1977) approach is sufficient for an initial estimate of the tidally-induced terms in the external potential, but it neglects terms which are of the order of the product of the tidal perturbation and the rotational perturbation. Here we demonstrate that, for a rapidly-rotating giant planet, the latter terms make a significant contribution to the love numbers $k_{n m}$, as well as (unobservably small) tidal contributions to the gravitational moments $J_{n}$.

Vorontsov et al. (1984) introduced a novel approach to calculation of the tidal response of giant planets. Rather than treating the problem as a purely static one, as we do here, they considered the case of a non-rotating giant planet orbited by a single satellite with an inertial orbital frequency $\Omega_{s}$. They then calculated the response of the planet's normal oscillation modes to the perturbation, noting that the mode frequencies (whose oscillation periods are measured in hours) are much higher than satellite orbital frequencies (satellite periods are measured in days). For such off-resonance excitation, it is unnecessary to 85 consider damping (as parameterized by the tidal quality factor $Q$ ) in calculating the tidal response. Taking the limit $\Omega_{s} \rightarrow 0$, Vorontsov et al. (1984) obtained the static tidal response of the non-rotating planet and thus its love number $k_{2}$. We compare the Vorontsov et al. Saturn $k_{2}$ with our value in Section 4.2 . below.

An analogous problem has been studied for the tidal response of Galilean satellite Io by Zharkov (2004) and Zharkov and Gudkova (2010), and for close-in exoplanets by Correia and Rodríguez (2013). These works consider the second order approximations through a higher order perturbative theory. Our problem is different, however, in that the tidal and rotational perturbations for Io are of 95 comparable magnitude, while the large influence of rotation on a much weaker tidal response found here for Saturn is unlike Io. Similarly, close-in, tidally 
locked exoplanets have comparable tidal and rotational perturbations.

Folonier et al. (2015) presented a method for approximating the love numbers of a non-homogeneous body using Clairaut theory for the equilibrium ellipsoidal figures. This results in an expression for the love number $k_{2}$ for a body composed of concentric ellipsoids, parameterized by their flattening parameters. In the case of the constant density spheroid, there is a well-known result that the equipotential surface is an ellipsoid. However, in bodies with more complicated density distributions, the equipotential surfaces will have a more general spheroidal shape. Because of the small magnitude of tidal perturbations, the method of Folonier et al. (2015) works in the limit of slow rotation despite this limitation. However, the method does not account for the coupled effect of tides and rotation, and does not predict love numbers of order higher than $k_{2}$. Within these constraints, we show below that our extended CMS method yields results that are in excellent agreement with results from Folonier et al. (2015).

Although our theory is quite general and can be used to calculate a rotating planet's static tidal response to multiple satellites located at arbitrary latitudes, longitudes, and radial distances, for application to Jupiter and Saturn it suffices to consider the effect of a single perturbing satellite sitting on an orbital plane at zero inclination to the planet's equator. Since tidal distortions are always very small compared with rotational distortion, and Jupiter's Galilean satellites, as well many of Saturn's larger satellites, are on orbits with low inclination, the tidal response to multiple satellites can be obtained by a linear superposition of the perturbation from each body. Extension of our theory to a system with a large satellite on an inclined orbit, such as Neptune-Triton, would be straightforward, but is not considered here. 


\section{Concentric Maclaurin Spheroid method with tides}

\subsection{Model parameters}

In the co-rotating frame of the planet in hydrostatic equilibrium, the pressure $P$, the mass density $\rho$ and the total effective potential $U$ are related by

$$
\nabla P=\rho \nabla U .
$$

The total effective potential can be separated into three components,

$$
U=V+Q+W
$$

where $V$ is the gravitational potential arising from the mass distribution within the planet, $Q$ is the centrifugal potential corresponding to a rotation frequency $\omega$, and $W$ is the tidal potential arising from a satellite with mass $m_{\mathrm{s}}$ at planetcentered coordinates $\left(R, \mu_{s}, \phi_{s}\right)$, where $R$ is the satellite's orbital distance from the origin, $\mu_{s}=\cos \theta$, where $\theta$ is the satellite's planet-centered colatitude and $\phi_{s}$ is the planet-centered longitude. In this investigation, we treat only the static tides in the corotating frame of the planet, and thus we always place the satellite at angular coordinates $\mu_{s}=0$ and $\phi_{s}=0$. The relative magnitudes of $V, Q$, and $W$ can be described in terms of two non-dimensional numbers:

$$
q_{\mathrm{rot}}=\frac{\omega^{2} a^{3}}{G M}
$$

for the rotational perturbation and

$$
q_{\mathrm{tid}}=-\frac{3 m_{\mathrm{s}} a^{3}}{M R^{3}}
$$

for the tidal perturbation, where $G$ is the universal gravitational constant, and $M$ and $a$ are the mass and maximum equatorial radius of the planet. The planet-satellite system is described by these two small parameters along with a third parameter, the ratio $a / R$.

Since CMS theory is nonperturbative, in principle our results are valid to all powers of these small parameters and their products (until we reach the computer's numerical precision limit). For the giant-planet tidal problems that we 
consider here, terms of second and higher order in $q_{\mathrm{tid}}$ are always negligible, but terms linear in $q_{\mathrm{tid}}$ and multiplied by various powers of $q_{\mathrm{rot}}$ and $a / R$ contribute above the numerical noise level. It is, in fact, terms of order $q_{\text {tid }} \cdot q_{\text {rot }}$ that contribute most importantly to the new results of this paper.

We introduce dimensionless planetary units of pressure $P_{\mathrm{pu}}$, density $\rho_{\mathrm{pu}}$, and total potential $U_{\mathrm{pu}}$, such that

$$
\begin{aligned}
P & \equiv \frac{G M^{2}}{a^{4}} P_{\mathrm{pu}} \\
\rho & \equiv \frac{M}{a^{3}} \rho_{\mathrm{pu}} \\
U & \equiv \frac{G M}{a} U_{\mathrm{pu}} .
\end{aligned}
$$

The CMS method considers a model planet composed of $N$ nested spheroids of constant density as depicted in Figure 1 . We label these spheroids with index $i=0,1,2, \ldots, N-1$, with $i=0$ corresponding to the outermost spheroid and $i=N-1$ corresponding to the innermost spheroid. Each spheroid is constrained to have a point at radial distance $a_{i}$ from the planet's center of mass, such that each of these fixed points has the same angular coordinates as the sub-satellite point ( $\mu=0, \phi=0)$. Accordingly, the $a_{0}$ of the outermost spheroid corresponds to its the largest principal axis, if the perturbing satellite is in the equatorial plane.

When $q_{\mathrm{tid}}=0$, the potential is axially symmetric and the problem can be solved in two spatial dimensions. However, when both $q_{\text {tid }}$ and $q_{\text {rot }}$ are nonzero, the symmetry is broken, meaning that each spheroid has a fully triaxial figure with the surface described by

$$
\zeta_{i} \equiv r_{i}(\mu, \phi) / a_{i}
$$

such that $\zeta_{0}$ represents the shape of the outer surface.

Taking advantage of the principle of superposition for a linear relationship between the potential $V$ and the mass density $\rho$, the total $V$ is given by the sum of the potential arising from each individual spheroid (Hubbard, 2013). This allows us to approximate any monotonically increasing density profile, with the 
density of the $i$ th spheroid represented by the density jump

$$
\delta \rho_{i}= \begin{cases}\rho_{i}-\rho_{i-1}, & i>0 \\ \rho_{0}, & i=0 .\end{cases}
$$

This parameterization of density has the added benefit of naturally handling discontinuities in $\rho$, as would be expected for a giant planet with a dense central core.

\subsection{Calculation of gravitational potential}

The general expansion of $V$ in spherical coordinates $\mathbf{r}=(r, \mu=\cos \theta, \phi)$ is

$$
\begin{aligned}
& V(r, \mu, \phi)= \\
& \frac{G}{r}\left[\sum_{n=0}^{\infty} P_{n}(\mu) \int_{\tau} d \tau \rho\left(r^{\prime}\right) P_{n}\left(\mu^{\prime}\right)\left(\frac{r^{\prime}}{r}\right)^{k}\right. \\
& +\sum_{n=0}^{\infty} \sum_{m=1}^{n} P_{n}^{m}(\mu) \cos (m \phi) \int_{\tau} d \tau \frac{2(n-m) !}{(n+m) !} \rho\left(r^{\prime}\right) P_{n}^{m}\left(\mu^{\prime}\right) \cos \left(m \phi^{\prime}\right)\left(\frac{r^{\prime}}{r}\right)^{k} \\
& \left.+\sum_{n=0}^{\infty} \sum_{m=1}^{n} P_{n}^{m}(\mu) \sin (m \phi) \int_{\tau} d \tau \frac{2(n-m) !}{(n+m) !} \rho\left(r^{\prime}\right) P_{n}^{m}\left(\mu^{\prime}\right) \sin \left(m \phi^{\prime}\right)\left(\frac{r^{\prime}}{r}\right)^{k}\right]
\end{aligned}
$$

(Zharkov and Trubitsyn, 1978), where $P_{n}$ and $P_{n}^{m}$ are the Legendre and associated Legendre polynomials,

$$
d \tau=r^{\prime 2} d r^{\prime} \sin \left(\theta^{\prime}\right) d \theta^{\prime} d \phi^{\prime}=r^{\prime 2} d r^{\prime} d \mu^{\prime} d \phi^{\prime},
$$

and the origin, $\mathbf{r}=(0,0,0)$, is the center of mass of the planet. The potential at a general point within the planet has a contribution from mass both interior and exterior to that point, for which the exponent $k$ in Eqn. (8) is different:

$$
k= \begin{cases}n, & r^{\prime}<r \\ -(n+1), & r^{\prime}>r .\end{cases}
$$

The centrifugal potential $Q$ depends only on $r$ and $\mu$

$$
Q(r, \mu)=\frac{1}{3} r^{2} \omega^{2}\left[1-P_{2}(\mu)\right]
$$


The tidal potential $W$ for a satellite at position $\mathbf{R}=\left(R, \mu_{s}, \phi_{s}\right)$ is

$$
W(\mathbf{r})=\frac{G m_{\mathrm{s}}}{|\mathbf{R}-\mathbf{r}|} .
$$

The general expansion of $W$ around the center of mass of the planet is obtained by using the summation theorem for spherical harmonics (Gavrilov and Zharkov 1977)

$$
\begin{aligned}
W(r, \mu, \phi)= & \frac{G m_{\mathrm{s}}}{R} \sum_{n=2}^{\infty}\left(\frac{r}{R}\right)^{n}\left[P_{n}(\mu) P_{n}\left(\mu_{s}\right)\right. \\
& \left.+2 \sum_{m=1}^{n} \frac{(n-m) !}{(n+m) !} \cos \left(m \phi-m \phi_{s}\right) P_{n}^{m}(\mu) P_{n}^{m}\left(\mu_{s}\right)\right] .
\end{aligned}
$$

Following Hubbard (2013), we derive non-dimensional quantities in terms of the planet mass $M$ and maximum radius $a=a_{0}$. For each spheroid, we define a dimensionless radius of each spheroid

$$
\lambda_{i} \equiv a_{i} / a
$$

and dimensionless density increment, based on the mean density of the planet

$$
\begin{aligned}
\bar{\rho} & =\frac{3 M}{a^{3}} \frac{1}{\int_{-1}^{1} d \mu^{\prime} \int_{0}^{2 \pi} d \phi^{\prime} \zeta_{0}^{3}} \\
\delta_{i} & \equiv \frac{\delta \rho_{i}}{\bar{\rho}} .
\end{aligned}
$$

A non-dimensional mass of the planet is then given by the integral expression

$$
M^{*}=\frac{1}{3} \sum_{j=0}^{N-1} \delta_{j} \lambda_{j}^{3} \int_{-1}^{1} d \mu^{\prime} \int_{0}^{2 \pi} d \phi^{\prime} \zeta_{j}^{3},
$$

which is equal to unity when $\delta_{j}$ is properly normalized for $\zeta_{j}$. The contribution to the potential is expanded in terms of interior and external zonal harmonics $J_{i, n}$ and $J_{i, n}^{\prime}$. For the tidal problem, we must also consider the analogous $C_{i, n m}, C_{i, n m}^{\prime}, S_{i, n m}$ and $S_{i, n m}^{\prime}$ (Zharkov and Trubitsyn, 1978). These contribute linearly to the total moment evaluated exterior to the planet's surface

$$
J_{n}=\sum_{i=0, N-1} J_{i, n}
$$


The layer-specific harmonics are then normalized by radius as

$$
\begin{array}{rlrl}
\widetilde{J}_{i, n} & \equiv \frac{J_{i, n}}{\lambda_{i}^{n}}, & \widetilde{J}_{i, n}^{\prime} \equiv J_{i, n}^{\prime} \lambda_{i}^{(n+1)} \\
\widetilde{S}_{i, n m} & \equiv \frac{S_{i, n m}}{\lambda_{i}^{n}}, & \widetilde{S}_{i, n m}^{\prime} \equiv S_{i, n m}^{\prime} \lambda_{i}^{(n+1)} \\
\widetilde{C}_{i, n m} \equiv \frac{C_{i, n m}}{\lambda_{i}^{n}}, & \widetilde{C}_{i, n m}^{\prime} \equiv C_{i, n m}^{\prime} \lambda_{i}^{(n+1)} .
\end{array}
$$

Following the derivation in Hubbard (2013) and generalizing the expressions for full three dimensional volume integrals, we find the normalized interior harmonics

$$
\begin{aligned}
& \widetilde{J}_{i, n}=\quad-\frac{3}{n+3} \frac{\delta_{i} \lambda_{i}^{3} \int_{-1}^{1} d \mu^{\prime} P_{n}\left(\mu^{\prime}\right) \int_{0}^{2 \pi} d \phi^{\prime} \zeta_{i}^{(n+3)}}{\sum_{j=0}^{N-1} \delta_{j} \lambda_{j}^{3} \int_{-1}^{1} d \mu^{\prime} \int_{0}^{2 \pi} d \phi^{\prime} \zeta_{j}^{3}} \\
& \widetilde{C}_{n m}=\frac{6(n-m) !}{(n+3)(n+m) !} \frac{\delta_{i} \lambda_{i}^{3} \int_{-1}^{1} d \mu^{\prime} P_{n}^{m}\left(\mu^{\prime}\right) \int_{0}^{2 \pi} d \phi^{\prime} \zeta_{i}^{(n+3)} \cos \left(m \phi^{\prime}\right)}{\sum_{j=0}^{N-1} \delta_{j} \lambda_{j}^{3} \int_{-1}^{1} d \mu^{\prime} \int_{0}^{2 \pi} d \phi^{\prime} \zeta_{j}^{3}} \\
& \widetilde{S}_{n m}=\frac{6(n-m) !}{(n+3)(n+m) !} \frac{\delta_{i} \lambda_{i}^{3} \int_{-1}^{1} d \mu^{\prime} P_{n}^{m}\left(\mu^{\prime}\right) \int_{0}^{2 \pi} d \phi^{\prime} \zeta_{i}^{(n+3)} \sin \left(m \phi^{\prime}\right)}{\sum_{j=0}^{N-1} \delta_{j} \lambda_{j}^{3} \int_{-1}^{1} d \mu^{\prime} \int_{0}^{2 \pi} d \phi^{\prime} \zeta_{j}^{3}},
\end{aligned}
$$

and the exterior harmonics

$$
\begin{aligned}
\widetilde{J}_{i, n}^{\prime} & = \\
\widetilde{C}_{n m}^{\prime} & =\frac{6(n-m) !}{(2-n)(n+m) !} \frac{\delta_{i} \lambda_{i}^{3} \int_{-1}^{1} d \mu^{\prime} P_{n}^{m}\left(\mu^{\prime}\right) \int_{0}^{2 \pi} d \phi^{\prime} \zeta_{i}^{(-n+2)} \cos \left(m \phi^{\prime}\right)}{\sum_{i}^{3} \int_{-1}^{1} d \mu^{\prime} P_{n}\left(\mu^{\prime}\right) \int_{0}^{2 \pi} d \phi^{\prime} \zeta_{i}^{(-n+2)}} \\
\widetilde{S}_{j=0}^{N-1} \delta_{j} \lambda_{j}^{3} \int_{-1}^{1} d \mu^{\prime} \int_{0}^{2 \pi} d \phi^{\prime} \zeta_{j}^{3} & =\frac{6(n-m) !}{(2-n)(n+m) !} \frac{\delta_{i} \lambda_{i}^{3} \int_{-1}^{1} d \mu^{\prime} P_{n}^{m}\left(\mu^{\prime}\right) \int_{0}^{2 \pi} d \phi^{\prime} \zeta_{i}^{(-n+2)} \sin \left(m \phi^{\prime}\right)}{\sum_{j=0}^{N-1} \delta_{j} \lambda_{j}^{3} \int_{-1}^{1} d \mu^{\prime} \int_{0}^{2 \pi} d \phi^{\prime} \zeta_{j}^{3}}
\end{aligned}
$$

with a special case for $n=2$

$$
\begin{array}{lr}
\widetilde{J}_{i, n}^{\prime}= & -3 \frac{\delta_{i} \lambda_{i}^{3} \int_{-1}^{1} d \mu^{\prime} P_{n}\left(\mu^{\prime}\right) \int_{0}^{2 \pi} d \phi^{\prime} \log \left(\zeta_{i}\right)}{\sum_{j=0}^{N-1} \delta_{j} \lambda_{j}^{3} \int_{-1}^{1} d \mu^{\prime} \int_{0}^{2 \pi} d \phi^{\prime} \zeta_{j}^{3}} \\
\widetilde{C}_{n m}^{\prime}= & \frac{6(n-m) !}{(n+m) !} \frac{\delta_{i} \lambda_{i}^{3} \int_{-1}^{1} d \mu^{\prime} P_{n}^{m}\left(\mu^{\prime}\right) \int_{0}^{2 \pi} d \phi^{\prime} \log \left(\zeta_{i}\right) \cos \left(m \phi^{\prime}\right)}{\sum_{j=0}^{N-1} \delta_{j} \lambda_{j}^{3} \int_{-1}^{1} d \mu^{\prime} \int_{0}^{2 \pi} d \phi^{\prime} \zeta_{j}^{3}} \\
\widetilde{S}_{n m}^{\prime}= & \frac{6(n-m) !}{(n+m) !} \frac{\delta_{i} \lambda_{i}^{3} \int_{-1}^{1} d \mu^{\prime} P_{n}^{m}\left(\mu^{\prime}\right) \int_{0}^{2 \pi} d \phi^{\prime} \log \left(\zeta_{i}\right) \sin \left(m \phi^{\prime}\right)}{\sum_{j=0}^{N-1} \delta_{j} \lambda_{j}^{3} \int_{-1}^{1} d \mu^{\prime} \int_{0}^{2 \pi} d \phi^{\prime} \zeta_{j}^{3}}
\end{array}
$$

and

$$
J_{i, 0}^{\prime \prime}=\frac{2 \pi \delta_{i} a_{0}^{3}}{3 M} .
$$


The shape of the surface of the planet is defined by the equipotential relationship

$$
U\left(\zeta, \mu, \phi, \mu_{s}, \phi_{s}\right)-U\left(1,0,0, \mu_{s}, \phi_{s}\right)=0,
$$

where the potential in planetary units at an arbitrary point on the planet's surface

$$
\begin{aligned}
U\left(\zeta, \mu, \phi, \mu_{s}, \phi_{s}\right)= & \frac{1}{\zeta_{0}}\left[1-\sum_{i=0}^{N-1} \sum_{n=1}^{\infty} \lambda_{i}^{n} \zeta_{0}^{-n}\left\{P_{n}(\mu) \widetilde{J}_{i, n}\right.\right. \\
& \left.-\sum_{m=1}^{n} P_{n}^{m}(\mu)\left(\widetilde{C}_{i, n m} \cos (m \phi)+\widetilde{S}_{i, n m} \sin (m \phi)\right)\right\} \\
& +\frac{1}{3} q_{\mathrm{rot}} \zeta_{0}^{3}\left[1-P_{2}(\mu)\right] \\
& -\frac{1}{3} \zeta_{0}^{3} q_{\mathrm{tid}} \sum_{n=2}^{\infty}\left(\frac{a}{R}\right)^{(n-2)} \zeta_{0}^{(n-2)}\left\{P_{n}(\mu) P_{n}\left(\mu_{s}\right)\right. \\
& \left.\left.+2 \sum_{m=1}^{n} \frac{(n-m) !}{(n+m) !} \cos \left(m \phi-m \phi_{s}\right) P_{n}^{m}(\mu) P_{n}^{m}\left(\mu_{s}\right)\right\}\right]
\end{aligned}
$$

matches the reference potential at the sub-satellite point

$$
\begin{aligned}
U\left(1,0,0, \mu_{s}, \phi_{s}\right)= & -\sum_{i=0}^{N-1} \sum_{n=1}^{\infty} \lambda_{i}^{n}\left\{P_{n}(0) \widetilde{J}_{i, n}-\sum_{m=1}^{n} P_{n}^{m}(0) \widetilde{C}_{i, n m}\right\} \\
& +\frac{1}{2} q_{\mathrm{rot}}-\frac{1}{3} q_{\mathrm{tid}} \sum_{n=2}^{\infty}\left(\frac{a}{R}\right)^{(n-2)}\left\{P_{n}(0) P_{n}\left(\mu_{s}\right)\right. \\
& \left.+2 \sum_{m=1}^{n} \frac{(n-m) !}{(n+m) !} \cos \left(-m \phi_{s}\right) P_{n}^{m}(0) P_{n}^{m}\left(\mu_{s}\right)\right\} .
\end{aligned}
$$

Similarly, the shapes of the interior spheroids are found by solving

$$
U_{j}\left(\zeta, \mu, \phi, \mu_{s}, \phi_{s}\right)-U_{j}\left(1,0,0, \mu_{s}, \phi_{s}\right)=0,
$$


where

$$
\begin{aligned}
U_{j}\left(\zeta_{j}, \mu, \phi, \mu_{s}, \phi_{s}\right)= & -\frac{1}{\zeta_{j} \lambda_{j}}\left[\sum _ { i = j } ^ { N - 1 } \sum _ { n = 0 } ^ { \infty } ( \frac { \lambda _ { i } } { \lambda _ { j } } ) ^ { n } \zeta _ { j } ^ { - n } \left\{P_{n}(\mu) \widetilde{J}_{i, n}\right.\right. \\
& \left.-\sum_{m=1}^{n} P_{n}^{m}(\mu)\left(\widetilde{C}_{i, n m} \cos (m \phi)+\widetilde{S}_{i, n m} \sin (m \phi)\right)\right\} \\
& +\sum_{i=0}^{j-1} \sum_{n=0}^{\infty}\left(\frac{\lambda_{j}}{\lambda_{i}}\right)^{n+1} \zeta_{j}^{n+1}\left\{\widetilde{J}_{i, n}^{\prime} P_{n}(\mu)\right. \\
& \left.-\sum_{m=1}^{n} P_{n}^{m}(\mu)\left(\widetilde{C}_{i, n m}^{\prime} \cos (m \phi)+\widetilde{S}_{i, n m}^{\prime} \sin (m \phi)\right)\right\} \\
& \left.+\sum_{i=0}^{j-1} J_{i, 0}^{\prime \prime} \lambda_{j}^{3} \zeta_{j}^{3}\right]+\frac{1}{3} q_{\mathrm{rot}} \lambda_{j}^{2} \zeta_{j}^{2}\left[1-P_{2}(\mu)\right] \\
& -\frac{1}{3} \lambda_{j}^{2} \zeta_{j}^{2} q_{\mathrm{tid}} \sum_{n=2}^{\infty}\left(\frac{a \lambda_{j}}{R}\right)^{(n-2)} \zeta_{j}^{(n-2)}\left\{P_{n}(\mu) P_{n}\left(\mu_{s}\right)\right. \\
& \left.+2 \sum_{m=1}^{n} \frac{(n-m) !}{(n+m) !} \cos \left(m \phi-m \phi_{s}\right) P_{n}^{m}(\mu) P_{n}^{m}\left(\mu_{s}\right)\right\}
\end{aligned}
$$

and

$$
\begin{aligned}
U_{j}\left(1,0,0, \mu_{s}, \phi_{s}\right)= & -\frac{1}{\lambda_{j}}\left[\sum_{i=j}^{N-1} \sum_{n=0}^{\infty}\left(\frac{\lambda_{i}}{\lambda_{j}}\right)^{n}\left\{P_{n}(0) \widetilde{J}_{i, n}-\sum_{m=1}^{n} P_{n}^{m}(0) \widetilde{C}_{i, n m}\right\}\right. \\
& +\sum_{i=0}^{j-1} \sum_{n=0}^{\infty}\left(\frac{\lambda_{j}}{\lambda_{i}}\right)^{n+1}\left\{\widetilde{J}_{i, n}^{\prime} P_{n}(0)-\sum_{m=1}^{n} P_{n}^{m}(0) \widetilde{C}_{i, n m}^{\prime}\right\} \\
& \left.+\sum_{i=0}^{j-1} J_{i, 0}^{\prime \prime} \lambda_{j}^{3}\right]+\frac{1}{2} q_{\mathrm{rot}} \lambda_{j}^{2} \\
& -\frac{1}{3} \lambda_{j}^{2} q_{\mathrm{tid}} \sum_{n=2}^{\infty}\left(\frac{a \lambda_{j}}{R}\right)^{(n-2)}\left\{P_{n}(0) P_{n}\left(\mu_{s}\right)\right. \\
& \left.+2 \sum_{m=1}^{n} \frac{(n-m) !}{(n+m) !} \cos \left(-m \phi_{s}\right) P_{n}^{m}(0) P_{n}^{m}\left(\mu_{s}\right)\right\} .
\end{aligned}
$$

From Eqn. (26), we also find the potential at the center of the planet

$$
U_{\text {center }}=-\sum_{i=0}^{N-1} \sum_{n=0}^{\infty} \lambda_{i}\left\{\widetilde{J}_{i, n}^{\prime}-\sum_{m=1}^{n} \widetilde{C}_{i, n m}^{\prime}\right\} .
$$


Taking the limit of Eqn. (27) as the radius goes to zero yields

$$
\begin{aligned}
U_{\text {center }} & =\lim _{\zeta_{j} \rightarrow 0} U_{j}\left(\zeta_{j}\right) \\
& =-\sum_{i=0}^{N-1} \frac{J_{i, n=0}^{\prime}}{\lambda_{i}},
\end{aligned}
$$

correcting a typographical error in Eqn. 49 of Hubbard (2013). In solving equations (21) and (24), we also require their analytical derivatives

$$
\begin{aligned}
\frac{d\left[U\left(\zeta, \mu, \phi, \mu_{s}, \phi_{s}\right)-U\left(1,0,0, \mu_{s}, \phi_{s}\right)\right]}{d \zeta} & =\frac{d U(\zeta, \mu, \phi)}{d \zeta} \\
\frac{d\left[U_{j}\left(\zeta_{j}, \mu, \phi, \mu_{s}, \phi_{s}\right)-U_{j}\left(1,0,0, \mu_{s}, \phi_{s}\right)\right]}{d \zeta_{j}} & =\frac{d U_{j}\left(\zeta_{j}, \mu, \phi\right)}{d \zeta_{j}} .
\end{aligned}
$$

\subsection{Gaussian quadrature} pre-calculate the values of all of the Legendre and associated Legendre polynomials at each polar quadrature point, $P_{n}\left(\mu_{\alpha}\right)$ and $P_{n}^{m}\left(\mu_{\alpha}\right)$.

For the azimuthal angle, we encounter integrals of the form

$$
\begin{aligned}
I_{c, m} & \equiv \int_{0}^{2 \pi} f(\phi) \cos (m \phi) d \phi \\
I_{s, m} & \equiv \int_{0}^{2 \pi} f(\phi) \sin (m \phi) d \phi
\end{aligned}
$$


when calculating the tesseral harmonics. For these, we use Chebyshev-Gauss integration with $L_{2}=96$ quadrature points $\eta_{\beta}=\cos \left(\phi_{\beta}\right), \beta=1,2, \ldots L_{2}$, with the corresponding weights $\omega_{\beta}, \beta=1,2, \ldots L_{2}$ over the interval $0<\phi<2 \pi$

$$
\begin{aligned}
d \eta & =-\sin (\phi) d \phi \\
d \phi & =-\frac{d \eta}{\sqrt{1-\eta^{2}}} .
\end{aligned}
$$

Using the identity $(\sin \theta)^{m-k}=\left(1-\mu^{2}\right)^{\frac{m-k}{2}}$, the sinusoidal functions can be expanded as

$$
\begin{aligned}
\cos m \phi & =\sum_{k=0}^{m}\left(\begin{array}{c}
m \\
k
\end{array}\right) \eta^{k}\left(1-\eta^{2}\right)^{\frac{m-k}{2}} \cos \left\{\frac{\pi}{2}(m-k)\right\} \\
\sin m \phi & =\sum_{k=0}^{m}\left(\begin{array}{c}
m \\
k
\end{array}\right) \eta^{k}\left(1-\eta^{2}\right)^{\frac{m-k}{2}} \sin \left\{\frac{\pi}{2}(m-k)\right\} .
\end{aligned}
$$

Substituting these into Eqn. 300 and splitting the integral into two intervals $0<\phi<\pi$ and $\pi<\phi<2 \pi$ yields

$$
\begin{aligned}
I_{c, m}= & \sum_{k=0}^{m}\left(\begin{array}{c}
m \\
k
\end{array}\right) \cos \left[\frac{\pi}{2}(m-k)\right]\left\{\int_{-1}^{1} \eta^{k} f\left(\cos ^{-1}(-\eta)\right)\left[1-\eta^{2}\right]^{\frac{m-k}{2}} d \eta\right. \\
& \left.-\int_{-1}^{1} \eta^{k} f\left(\cos ^{-1} \eta\right)\left[1-\eta^{2}\right]^{\frac{m-k}{2}} d \eta\right\} \\
= & \sum_{k=0}^{m}\left(\begin{array}{c}
m \\
k
\end{array}\right) \cos \left[\frac{\pi}{2}(m-k)\right] \\
& *\left\{ \pm \sum_{\beta=1}^{L_{2}} \omega_{\beta} \eta_{\beta}^{k} f\left(\pi-\cos ^{-1}\left(\eta_{\beta}\right)\right)\left[1-\eta_{\beta}^{2}\right]^{\frac{m-k}{2}}\right. \\
& \left.-\sum_{\beta=1}^{L_{2}} \omega_{\beta} \eta_{\beta}^{k} f\left(\cos ^{-1} \eta_{\beta}\right)\left[1-\eta_{\beta}^{2}\right]^{\frac{m-k}{2}}\right\},
\end{aligned}
$$

where the sign of the second sum depends on the parity of $m$. When calculating the zonal harmonics, the integral $I_{c, m}\left(f\left(\mu_{\alpha}, \phi_{\beta}\right)\right)$ reduces to the axisymmetric solution with $m=0$. The zonal harmonics Eqn. (17) can, therefore, be calculated via the summation

$$
\widetilde{J}_{i, n} \approx-\left(\frac{3}{n+3}\right)\left(\frac{\delta_{i} \lambda_{i}^{3} \sum_{\alpha=1}^{L_{1}} \omega_{\alpha} P_{n}\left(\mu_{\alpha}\right) I_{c, 0}\left(\zeta_{i \alpha \beta}^{(n+3)}\right)}{\sum_{j=0}^{N-1} \delta_{j} \lambda_{j}^{3} \sum_{\alpha=1}^{L_{1}} \omega_{\alpha} I_{c, 0}\left(\zeta_{j \alpha \beta}^{3}\right)}\right)
$$


and the tesseral harmonics likewise via

$$
\widetilde{C}_{n m} \approx \frac{6(n-m) !}{(n+3)(n+m) !}\left(\frac{\delta_{i} \lambda_{i}^{3} \sum_{\alpha=1}^{L_{1}} \omega_{\alpha} P_{n}^{m}\left(\mu_{\alpha}\right) I_{c, m}\left(\zeta_{i \alpha \beta}^{(n+3)}\right)}{\sum_{j=0}^{N-1} \delta_{j} \lambda_{j}^{3} \sum_{\alpha=1}^{L_{1}} \omega_{\alpha} I_{c, 0}\left(\zeta_{j \alpha \beta}^{3}\right)}\right) .
$$

There are analogous expressions for $I_{s, m}$ and $S_{n m}$, but these evaluate to zero in all calculations presented here due to the symmetry of the model.

\subsection{Iterative procedure}

We begin with initial estimates for the shape of each surface $\zeta_{i \alpha \beta, 0}$ and for the moments $\widetilde{J}_{i, n}, \widetilde{J}_{i, n}^{\prime}, \widetilde{J}_{i}^{\prime \prime}, \widetilde{C}_{i, n m}, \widetilde{C}_{i, n m}^{\prime}, \widetilde{S}_{i, n m}$, and $\widetilde{S}_{i, n m}^{\prime}$. For each iteration $t$ the level surfaces are then updated using a single Newton-Raphson integration step.

$$
\zeta_{i \alpha \beta, t+1}=\zeta_{i \alpha \beta, t}-\frac{f\left(\zeta_{i \alpha \beta, t}\right)}{f^{\prime}\left(\zeta_{i \alpha \beta, t}\right)}
$$

where $f$ is the equipotential relation, Equations 21 - 23 for the outermost surface and Equations $24-26$ for interior layers, and $f^{\prime}$ is the first derivative of that function with respect to $\zeta$, Eqn. (29). The multipole moments are then calculated for the updated $\zeta_{i \alpha \beta}$ via Equations $(17)-(19)$. These two steps are repeated until all of the exterior moments, $J_{n}, C_{n m}$ and $S_{n m}$, have converged such that the difference between successive iterations falls below a specified tolerance. Starting with a naive guess for the initial state, a typical calculation achieves a precision much higher than would be required for comparison with Juno measurements after about 40 iterations.

In simulations with a finite $q_{\mathrm{rot}}$ and $q_{\mathrm{tid}}$, we typically find an initial converged equilibrium shape with a non-zero, first-order harmonic coefficient $C_{11}$ of the order of $q_{\text {rot }} \cdot q_{\mathrm{tid}}$ or smaller. This indicates that the center of mass of the system is shifted slightly along the planet-satellite axis from the origin of the initial coordinate system. To remove this term, we apply a translation to the shape function of $\Delta x=-a \cdot C_{11}$ in the direction of the satellite. This correction requires approximating the coordinates $\left(\mu^{\prime}, \phi^{\prime}\right)$ in the uncorrected frame that correspond to the quadrature points $\mu_{\alpha}$ and $\phi_{\beta}$ in the corrected frame, so that the correct shape $\zeta$ is integrated to find the moments in the corrected frame. 
For a value of $q_{\text {tid }}$ similar to the gas giants, this correction yields a body with $C_{11}$ on the order of the specified tolerance. For systems with a much larger $q_{\text {tid }}$ (of which there are none in our planetary system), this second-order effect might affect the precision of the calculation. The residual effect is below the numerical noise level for the Saturn models presented in this paper.

\subsection{Calculation of the barotrope}

We first calculate the density of each uniform layer; for the $j$ th layer we have

$$
\rho_{j, \mathrm{pu}}=\frac{\sum_{i=0}^{j} \delta_{i}}{\sum_{k=0}^{N-1} \delta_{k} \lambda_{k}^{3} \int_{-1}^{1} d \mu^{\prime} \int_{0}^{2 \pi} d \phi^{\prime} \zeta_{k}^{3}} .
$$

Using this expression, we calculate the total potential $U_{\mathrm{pu}}$ on the surface of each layer and at the center using Equations 23) and (26) - 27). Since the density is constant between interfaces, the hydrostatic equilibrium relation, Eqn. (1) is trivially integrated to obtain the pressure at the bottom of the $j$ th layer.

$$
P_{j, \mathrm{pu}}=P_{j-1, \mathrm{pu}}+\rho_{j-1, \mathrm{pu}}\left(U_{j, \mathrm{pu}}-U_{j-1, \mathrm{pu}}\right)
$$

After obtaining a converged hydrostatic-equilibrium model for $\mathrm{N}$ spheroids with the above array using the initial density profile $\delta_{j}$, one calculates the arrays $U_{j, \mathrm{pu}}$ and $P_{j, \mathrm{pu}}$. Next, one calculates an array of desired densities

$$
\rho_{j, \mathrm{pu}, \text { desired }}=\rho\left(\frac{1}{2}\left(P_{j+1}+P_{j}\right)\right),
$$

where $\rho(P)$ is the inverse of the adopted barotrope $P(\rho)$. Finding the difference between the desired densities of subsequent layers then gives a new array of $\delta_{j}$ for use in the next iteration. In our implementation, it is also necessary to scale these densities by a constant factor to obtain the correct total mass of the CMS model.

Self-gravity from the model's rotational and tidal deformation will cause a small change in the density profile from that expected for a spherical body. In practice, only relatively large changes in the shape of the body will cause a significant deviation in the density profile. Since $q_{\text {rot }} \gg q_{\mathrm{tid}}$, the influence 


\subsection{Spheroid of constant density}

The well-known special case of a single constant-density spheroid is an important test, because it has a closed form, analytical solution to the theory of figures (Tassoul, 2015). In the case of non-zero $q_{\text {rot }}$ it is conventionally referred to as the Maclaurin spheroid, as the Jeans spheroid for finite $q_{\mathrm{tid}}$, and the Roche spheroid in the general case. In equilibrium, the spheroid will have an ellipsoidal shape. In the limit of a low-amplitude tidal perturbation and zero rotation, the love number for all permitted $n$ is

$$
k_{n}=\frac{3}{2(n-1)}
$$

(Munk and MacDonald, 2009).

From our simulation results, we calculate the love numbers as

$$
k_{n m}=-\frac{2}{3} \frac{(n+m) !}{(n-m) !} \frac{C_{n m}}{P_{n}^{m}(0) q_{\mathrm{tid}}}\left(\frac{a}{R}\right)^{2-n} .
$$

For simulations with finite $q_{\mathrm{tid}}$ and $q_{\mathrm{rot}}=0$, we find our calculated $k_{n m}$ to be degenerate with $m$ in accordance with the analytical result. For a given value of $n$,

$$
k_{n m}= \begin{cases}0, & n \text { and } m \text { opposite parity } \\ \text { const, } & n \text { and } m \text { same parity. }\end{cases}
$$


Figure 2 shows the calculated $k_{n}$ for the non-rotating Jeans spheroid as a function of $q_{\text {tid }}$ up to order $n=6$, with $R / a$ taken to be that for Tethys and Saturn. For a small tidal perturbation, we find that $k_{n}$ approaches the analytical result of Eqn. 40 . Conversely, as $q_{\text {rot }}$ approaches unity from below, the love numbers diverge, with $k_{n}$ decreasing for $n \leq 3$ and increasing for $n>3$. The departure from the analytical solution becomes significant $\left(\left|\Delta k_{n}\right|>0.1\right)$ for $-q_{\mathrm{tid}}>10^{-3}$, whereas for values representative of the largest Saturnian satellites, $k_{2}$ matches the analytic value to within our numerical precision.

In general, the tidal response of a gas giant planet will not be a perturbation to a perfect sphere, but to a spheroidal shape dominated by rotational flattening. Therefore, simulation of the tidal response in the absence of rotation is not generally applicable to real gas giants. When we simulate a Roche spheroid with both finite $q_{\text {rot }}$ and $q_{\mathrm{tid}}$, we find a different behavior for $k_{n m}$ as defined by Eqn. (41). Figure 3 shows the calculated $k_{n m}$ for a spheroid with a constant $q_{\text {tid }}$ and a variable $q_{\text {rot }}$. When the magnitude of $q_{\text {rot }}$ is comparable to $q_{\mathrm{tid}}$, the tidal response matches the expected analytical result. However, for $q_{\text {rot }}>10^{-3}$, we can see that the degeneracy of $k_{n m}$ with $m$ is broken, and all permitted $k_{n m}$ deviate from the expected values. In other words, Eqn. 42 becomes

$$
\begin{cases}k_{n m}=0, & n \text { and } m \text { opposite parity } \\ k_{n m} \neq \text { const, } & n \text { and } m \text { same parity }\end{cases}
$$

and all permitted $k_{n m}$ deviate from the expected values. We also note that these deviations become pronounced earlier for the higher order $n$.

\subsection{Two-layered spheroid}

Proceeding to more complicated interior structures has proved challenging

for analytical or semi-analytical methods. Even the next simplest model with two constant-density layers does not have a closed form solution for arbitrary order $n$. Folonier et al. (2015) present an extension of Clairaut theory for a multi-layer planet under the approximation that the level surfaces are perfect ellipsoids. Under this approximation, they derive an analytic solution for the 
$k_{2}$ as a function of two ratios of properties of the two layers, $a_{1} / a$ and $\rho_{0} / \rho_{1}$. Table 1 shows a comparison of our calculated $k_{2}$ with the analytic result from Folonier et al. (2015) for a selection of parameters spanning a range of $a_{1} / a$ and $\rho_{0} / \rho_{1}$. All of our results using the CMS method differ from those using Clairaut 235 theory by less than $10^{-5}$. This provides an important test of the correctness of the interior potentials used in our approach. It also indicates that ellipsoids, while not exact, are a very good approximation for the degree 2 tidal response shape in the limit of very small $q_{\mathrm{tid}}$, and $q_{\text {rot }}=0$.

\subsection{Polytrope of index unity}

The polytrope of index unity defines a more realistic barotrope that also lends itself to semi-analytic analyses. It corresponds to the relation

$$
P=K \rho^{2}
$$

where the polytropic constant $K$ can be chosen to match the planet's physical parameters. For a non-rotating $n=1$ polytrope, the density distribution is given by

$$
\rho=\rho_{c} \frac{\sin \pi \lambda}{\pi \lambda}
$$

where $\rho_{c}$ is the density at the center of the planet. To obtain the first approximation of $\delta_{j}$, we differentiate Eqn. 45 by $\lambda$ :

$$
\frac{d\left(\rho / \rho_{c}\right)}{d \lambda}=\frac{\cos \pi \lambda}{\lambda}-\frac{\sin \pi \lambda}{\pi \lambda^{2}} .
$$

We then correct this profile to be consistent with the given $q_{\text {rot }}$ via the method introduced in Section 2.5. Scaling the densities to maintain the total mass of the planet has a straightforward interpretation for a polytropic barotrope, as it is equivalent to changing $K$.

For the constant-density Roche spheroid the lowest degree love number was

$$
k_{2}=\frac{3}{2}
$$


Considering only the linear response to a purely rotational perturbation, we define a general degree 2 linear response parameter $\Lambda_{2}$ as

$$
J_{2}=\Lambda_{2} q_{\text {rot }}
$$

Whereas $\Lambda_{2}=1 / 2$ for the Roche spheroid, for the polytrope of index unity the analytic result is (Hubbard, 1975)

$$
\Lambda_{2}=\left(\frac{5}{\pi^{2}}-\frac{1}{3}\right)
$$

Considering linear response only, one finds in general

$$
k_{2}=3 \Lambda_{2}
$$

valid in the limit $q_{\text {rot }} \ll 1$ and $q_{\text {tid }} \ll 1$, for any barotrope in hydrostatic equilibrium. Thus, for the polytrope of index unity in this limit,

$$
k_{2}=\frac{15}{\pi^{2}}-1=0.519817755 .
$$

We compare this to a CMS simulation of the $n=1$ polytrope model with 128 layers, $q_{\mathrm{rot}}=0, q_{\mathrm{tid}}=10^{-6}$, and Tethys' $R / a$. The simulation results agree with the expected relation $J_{2}=2 C_{22}$ to numerical precision, and yield $k_{2}=0.519775$. This provides a test of the multi-layer CMS approach subject to a tidal-only perturbation. The CMS result matches our Eqn. (51) benchmark to better than the precision with which we could measure this parameter using the Juno spacecraft. The small difference can be attributed to approximation of a continuous polytrope by 128 layers in the CMS simulation. Wisdom and Hubbard (2016) (Eqn. 15) show the relative discretization error of a CMS polytrope model to be $\sim 10^{-3}$ for $N=128$, roughly consistent with our calculated difference.

Similar to the calculations on the constant density spheroid in Section 3.1 we performed additional $N=128$ polytrope simulations with finite $q_{\text {tid }}$ and $q_{\text {rot }}=0$. Once again, we find our calculated $k_{n m}$ to be degenerate with $m$ for the tidal-only simulations, in agreement with Eqn. 42. Figure 4 shows the behavior of $k_{n}$ for $n \leq 6$ for these tidal-only polytrope simulations. We only present these results up to $q_{\mathrm{tid}} \sim 10^{-4}$, because above that value effects of the 

to the polytropic relation, as discussed in Section 2.5. We observe that realistic values for $q_{\text {tid }}$ have negligible effect on the tidal response. Even for the IoJupiter system, the effect of finite $q_{\mathrm{tid}}$ on $k_{n m}$ is near the numerical noise level. The general behavior is quite similar to the case of the single Jeans spheroid. the single spheroid case, with $k_{2}$ asymptoting to the analytic limit in Eqn. (51). Similar to the single spheroid, the behavior as $q_{\text {rot }}$ increases from zero sees $k_{n}$ decrease for $n \leq 3$ and increase for $n>3$. The deviation from the low $q_{\text {tid }}$ value is also less pronounced for the more realistic polytrope density less mass concentrated in the outer portion of the polytrope model.

Figure 5 shows the effect of variable $q_{\text {rot }}$ on polytrope models with constant $q_{\text {tid }}$. Once again, we find that $k_{n m}$ degeneracy with respect to $m$ breaks, in agreement with Eqn. (43), as $q_{\text {rot }}$ increases. Although the splitting of $k_{n m}$ is still significant at large values of $q_{\text {rot }} \sim 10^{-2}$ consistent with the rapidly-rotating gas giants. The shift in $k_{n m}$ shows a nearly linear increase in magnitude with increasing $q_{\text {rot }}$, with potentially observable increases in $k_{2}$ for both the ice giant and gas giant planets. The general behavior of $k_{n m}$ is very similar between and directions of all $k_{n m}$ up to $n=6$ are similar between the two cases. This indicates that the effect should be ubiquitous in all fast-spinning liquid bodies, and relatively insensitive to the density profile of the planet.

\section{Saturn's tidal response}

\subsection{Saturn interior models}

Lainey et al. (2016) present the first determination of the love number $k_{2}$ for a gas giant planet using a dataset of astrometric observations of Saturn's coorbital moons. Their observed value $k_{2}=0.390 \pm 0.024$ is much larger than the 
theoretical prediction of 0.341 by Gavrilov and Zharkov (1977). Here we present with a total of 128 layers, for which the CMS model has a discretization error (Wisdom and Hubbard, 2016) smaller than uncertainty in the observations of Saturn's $k_{2}$. 

6. with densities and radii adjusted to yield agreement with both observed $J_{2}$ and observed $J_{4}$ as given in Table 2 . With a zero-density outermost layer, this leaves two free paramters, making it the simplest model that can match $J_{2}$ and $J_{4}$ exactly.

Finally, we include a third model using a more realistic H-He equation of state based on DFT-MD simulations, following the preliminary Jupiter model of Hubbard and Militzer (2016) with a Saturn adiabat. This model has a density discontinuity (Fig. 6) at 0.76 Mbar where the Saturn adiabat crosses the HHe phase separation curve of Morales et al. (2009). This allows the $J_{2}$ and $J_{4}$ values to be fitted exactly, by changing the metallicities above and below the discontinuity. While still schematic, it is the most realistic of our Saturn models.

The two simple models, while not particularly realistic, capture the major features of Saturn's internal structure. It is well established that the details of Saturn's internal structure are largely degenerate, with a wide range of possible core sizes and densities adequately matching the few observational constraints (Kramm et al., 2011; Helled and Guillot, 2013, Nettelmann et al., 2013). The qualitative similarities between our single spheroid and polytrope simulations (Sections 3.1 and 3.3) indicate that the rotational enhancement of $k_{2}$ should be a robust prediction regardless of the particular details of the interior profile. A comparison between our polytrope plus core and four layer models provides another test of the sensitivity of $k_{2}$ to interior structure. We do not consider here the influence of differential rotation (Hubbard, 1982, Kong et al., 2013. Cao and Stevenson, 2015, Wisdom and Hubbard, 2016), which might have an influence on the gravitational response in comparison to the solid-body rotation considered here.

\subsection{Calculated $k_{2}$ for Saturn}

We take our baseline model to be the $N=128$ CMS core plus polytrope model with physical parameters fitted to Cassini observations. Figure 7 shows the calculated zonal harmonics $J_{n}$ up to order $n=30$. The even $J_{n}$ decrease 
$J_{n}$ is negative when $n$ is divisible by 4 , and positive otherwise. The calculated $J_{n}$ are essentially indistinguishable from those calculated for the rotation only case with the same $q_{\text {rot }}$, as is expected given $q_{\text {rot }} \gg q_{\text {tid }}$. We may estimate the maximum effect of differential rotation by considering the change of the calcuvalue $\left(q_{\text {rot }}=0.155303\right)$ to the Cassini value $\left(q_{\text {rot }}=0.151616\right)$, a relative decrease in $q_{\text {rot }}$ of about $2 \%$. From Table 3 , we see that this change increases $k_{2}$ by about $2 \%$ (holding $J_{2}$ fixed). The net effect of a deep-seated smooth variation of rotation rate from the Voyager value near the equator to the Cassini value near the pole would presumably be smaller, depending on how much mass is involved in the differential flow. Cao and Stevenson (2015) have shown that the effect of realistic deep flow patterns on low order zonal harmonics is small, but a more quantitative evaluation of their effect on Saturn's $k_{2}$ remains to be done.

Figure 8 shows the magnitude of $C_{n m}$ for the core plus polytrope model with Cassini rotation. Changing the number of layers, satellite parameters or the rotation rate to the Voyager value leads to a shift in the values, but the relative magnitudes and signs of $C_{n m}$ remain approximately the same. In the same figure, we also compare the $C_{n m}$ for a non-rotating planet having the same density profile $\rho\left(\lambda_{i}\right)$. Here we see significant shifts in the $C_{n m}$ magnitudes, although the signs remain the same. For the rotating model, $C_{n m}$ is similar for most points where $n=m$, but with magnitudes significantly larger when $m<n$. The only exception to this trend is $C_{31}$ which is lower for the rotating model. These results are all broadly consistent with the splitting of $k_{n m}$ observed for the polytrope in Section 3.3

Table 3 summarizes our calculated values for $k_{2}$ for several different models. The identifying labels "Cassini" and "Voyager" use the observed rotation rate from Jacobson et al. (2006), and Desch and Kaiser (1981) respectively, while "non-rotating" is a model with $q_{\text {rot }}=0$. The "non-rotating" model uses the 380 
been relaxed to be in equilibrium for zero rotation. It does, however, allow us to quantify the effect of rotation on the tidal response by comparison with the "Cassini" model. "Tethys" and "Dione" refer to models with the satellite parameters $q_{\mathrm{tid}}$ and $R / a$ corresponding to those satellites, whereas "no tide" is an analogous model with finite $q_{\text {rot }}$ only. " $N=128$ " uses the polytrope outer envelope with constant density inner core, whereas " $N=4$ " is the model which independently adjusts layer densities to match the observed $J_{2}$ and $J_{4}$. The "DFT-MD" models use the H-He equation of state from Hubbard and Militzer (2016) with $N=511$ layers.

All of the rotating models yields a calculated $k_{2}$ value matching the observation of Lainey et al. (2016) within their error bars. Our baseline model yields $k_{2}=0.4130$, while using the Voyager observations yields a value $\sim 0.008$ lower. We find that the difference between the $k_{2}$ values associated with the satellites Tethys and Dione is $\sim 0.0003$, well below the current sensitivity limit. Using the $\sim 2.5 \%$ higher "Voyager" rotation rate leads to a decrease of $\sim 0.01$ in $k_{2}$.

In Table 3 , we also show the calculated $J_{2}, J_{4}$ and $J_{6}$ following the convergence of the gravitational field in response to the tidal perturbation. For the core plus polytrope model, the rotation rate from Voyager is more consistent with the $J_{4}$ and $J_{6}$ from Jacobson et al. (2006). This doesn't necessarily mean that the Voyager rotation rate is more correct, just that it allows a better fit for our simplified density model. Nonetheless, our fitted gravitational moments are much closer to each other than to those from the pre-Cassini model of Gavrilov and Zharkov (1977).

In comparison to the other models, the outlier is the non-rotating model, which underestimates the $k_{2}$ by $\sim 9.4 \%$ compared to a rotating body with the same density distribution. This calculated enhancement accounts for most of the difference between the observation of $k_{2}=0.390 \pm .024$ (Lainey et al., 2016) and the classical theory result of 0.341 (Gavrilov and Zharkov, 1977). We attribute our non-rotating model's larger $k_{2}$ to our different interior model which matches 410 more recent constraints on Saturn's zonal gravitational moments $J_{2}-J_{6}$.

We note that the later theoretical prediction of 0.386 by Vorontsov et al. 
(1984) is also compatible with the observed $k_{2}$. Their method considers the effect of free-oscillations on the tidal response of giant planets. While our rotating models yield higher values of $k_{2}$ than Vorontsov et al. (1984) our "non-rotating" include a nonlinear response to rotation and tides. Thus, future high-precision measurements of the $k_{n m}$ of Jovian planets, say to better than $0.1 \%$, will be useful for constraining basic parameters such as the interior rotation rate of the planet, and may help to break the current degeneracy of interior density profiles. 


\section{Summary}

The non-perturbative CMS method for calculating a self-consistent shape and gravitational field of a static liquid planet has been extended to include

the largest contribution to the low-order tesseral harmonics measured by Juno and future spacecraft studies of the gas giants. This approach has been benchmarked against analytical results for the tidal response of the constant density Jeans/Roche spheroid, a two constant density layer model and the polytrope of index unity.

We highlight for the first time an important effect of rapid rotation on the tidal response of the gas giants. CMS simulations of the tidal response on bodies with large rotational flattening show significant deviation in the tesseral harmonics of the gravitational field as compared to simulations without rotation. $n>2$. Meanwhile, it leads to an observable enhancement in $k_{2}$ compared to a non-rotating model.

This rotational enhancement of the $k_{2}$ love number for a simplified interior model of Saturn agrees with the recent observational result (Lainey et al., ${ }_{460}$ 2016), which found $k_{2}$ to be much higher than previous predictions. Our predicted values of $k_{2}$ are robust for reasonable assumptions of interior structure, rotation rate and satellite parameters. The Juno spacecraft is expected to measure Jupiter's gravitational field to sufficiently high precision to measure lower order tesseral components arising from Jupiter's large moons, and we predict an analogous rotational enhancement of $k_{2}$ for Jupiter. Our high-precision tidal theory will be an important component of the search for non-hydrostatic terms in Jupiter's external gravity field.

\section{Acknowledgments}

This work was supported by NASA's Juno project. Sean Wahl and Burkhard 
and astrophysics research grant 1412646). We thank Isamu Matsuyama for helpful discussions regarding classical tidal theory. We thank V. N. Zharkov and another referee for their useful comments.

\section{References}

Archinal, B.A., A'Hearn, M.F., Bowell, E., Conrad, A., Consolmagno, G.J., Courtin, R., Fukushima, T., Hestroffer, D., Hilton, J.L., Krasinsky, G.A., Neumann, G., Oberst, J., Seidelmann, P.K., Stooke, P., Tholen, D.J., Thomas, P.C., Williams, I.P., 2011. Report of the IAU Working Group on Cartographic Coordinates and Rotational Elements: 2006. Celest. Mech. Dyn.

Cao, H., Stevenson, D.J., 2015. Gravity and Zonal Flows of Giant Planets:

口 From the Euler Equation to the Thermal Wind Equation , 1-9URL: http: //arxiv.org/abs/1508.02764, arXiv:1508.02764.

Correia, A.C.M., Rodríguez, A., 2013. on the Equilibrium Figure of Close-in 485 ¿ Planets and Satellites. Astrophys. J. 767, 128. URL: http://arxiv.org/ abs/1304.1425, doi:10.1088/0004-637X/767/2/128, arXiv:1304.1425.

Desch, M.D., Kaiser, M.L., 1981. Voyager measurement of the rotation period

q of Saturn's magnetic field. Geophys. Res. Lett. 8, 253-256. doi10.1029/ GL008i003p00253.

490 Folonier, H., Ferraz-Mello, S., Kholshevnikov, K.V., 2015. The flattenings of the layers of rotating planets and satellites deformed by a tidal potential.

口 Celest. Mech. Dyn. Astron. 122, 183-198. URL: http://arxiv.org/abs/ 1503.08051, doi $10.1007 /$ s10569-015-9615-6, arXiv: 1503.08051 .

Gavrilov, S.V., Zharkov, V.N., 1977. Love numbers of the giant planets. 495 I Icarus 32, 443-449. URL: http://linkinghub.elsevier.com/retrieve/ pii/001910357790015X, doi 10.1016/0019-1035(77)90015-X. 
Giampieri, G., Dougherty, M.K., Smith, E.J., Russell, C.T., 2006. A regular period for Saturn's magnetic field that may track its internal rotation. Nature 441, 62-64. doi:10.1038/nature04750

ter Galileo mission. Planet. Space Sci. 47, 1201-1210. URL: http:

a //linkinghub.elsevier.com/retrieve/pii/S0032063399000446, doi 10 . 1016/S0032-0633(99)00044-6.

Helled, R., Guillot, T., 2013. Interior Models of Saturn: Includ505 ing the Uncertainties in Shape and Rotation. Astrophys. J. 767,

113. URL: http://stacks.iop.org/0004-637X/767/i=2/a=113?key=

a. crossref.045d858be83734acdc0600277a318377, doi 10.1088/0004-637X/ $767 / 2 / 113$

Hubbard, W., 1975. Gravitational field of a rotating planet with a polytropic index of unity. Sov. Astron. 18, 621-624.

Hubbard, W., 1982. Effects of differential rotation on the gravitational n figures of Jupiter and Saturn. Icarus 52, 509-515. URL: http:

n//linkinghub.elsevier.com/retrieve/pii/0019103582900112, doi/10. 1016/0019-1035(82)90011-2.

Hubbard, W., Schubert, G., Kong, D., Zhang, K., 2014. On the convergence of the theory of figures. Icarus 242, 138-141. URL: http:

a //linkinghub.elsevier.com/retrieve/pii/S001910351400428X doi 10. $1016 / j$. icarus.2014.08.014.

Hubbard, W.B., 2012. High-Precision Maclaurin-Based Models 520 of Rotating Liquid Planets. Astrophys. J. 756, L15. URL:

1. http://stacks.iop.org/2041-8205/756/i=1/a=L15?key=crossref. 34b95153bc3fdfb844cab51abbdf75d3, doi:10.1088/2041-8205/756/1/L15

Hubbard, W.B., 2013. Concentric Maclaurin Spheroid Models of Rotating Liquid Planets. Astrophys. J. 768, 43. URL: 
http://stacks. iop.org/0004-637X/768/i=1/a=43?key=crossref . a31bd47c857111e805198695ba70780e, doi $10.1088 / 0004-637 \mathrm{X} / 768 / 1 / 43$.

Hubbard, W.B., Militzer, B., 2016. A Preliminary Jupiter Model. Astrophys. J. 820. URL: http://arxiv.org/abs/1602.05143, arXiv:1602.05143.

Jacobson, R.A., Antresian, P.G., Bordi, J.J., Criddle, K.E., Ionasescu, R., ${ }_{530}$ Jones, J.B., Mackenzie, R.a., Meek, M.C., Parcher, D., Pelletier, F.J., Owen, W.M., Roth, D.C., Roundhill, I.M., Stauch, J.R., 2006. The gravity field of the Saturnian system from staellites observations and spacecraft tracking data. Astrophys. J. 132, 2520-2526. doi:10.1086/508812.

Jeans, J.H., 2009. Problems of Cosmology and Stellar Dynamics. Cambridge University Press. URL: http://dx.doi.org/10.1017/CB09780511694417.

Kaspi, Y., 2013. Inferring the depth of the zonal jets on Jupiter and Saturn

1. from odd gravity harmonics. Geophys. Res. Lett. 40, 676-680. doi 10.1029/ 2012GL053873.

Kaspi, Y., Hubbard, W.B., Showman, A.P., Flierl, G.R., 2010. Gravitational signature of Jupiter's internal dynamics. Geophys. Res. Lett. 37,

a L01204. URL: http://doi.wiley.com/10.1029/2012GL053873, doi 10. 1029/2009GL041385.

Kong, D., Liao, X., Zhang, K., Schubert, G., 2013. Gravitational signature of rotationally distorted Jupiter caused by deep zonal winds. Icarus 545 226, 1425-1430. URL: http://linkinghub.elsevier.com/retrieve/pii/ S0019103513003540, doi $10.1016 /$ j.icarus.2013.08.016.

Kramm, U., Nettelmann, N., Redmer, R., Stevenson, D.J., 2011. Astrophysics On the degeneracy of the tidal Love number $k_{2}$ in multi-layer planetary models q : application to Saturn and GJ 436b. Astron. Astrophys. 18, 1-7. doi 10. 1051/0004-6361/201015803, arXiv:1101.0997.

Lainey, V., Jacobson, R.A., Tajeddine, R., Cooper, N.J., Robert, V., Tobie, G., Guillot, T., Mathis, S., 2016. New constraints on Saturn's interior 
from Cassini astrometric data URL: http://arxiv.org/abs/1510.05870, arXiv: 1510.05870.

Morales, M.A., Schwegler, E., Ceperley, D., Pierleoni, C., Hamel, S., Caspersen, K., 2009. Phase separation in hydrogen-helium mixtures at a Mbar pressures. Proc. Natl. Acad. Sci. U. S. A. 106, 1324-9. URL: http: प //www.pubmedcentral.nih.gov/articlerender.fcgi?artid=2631077\& tool=pmcentrez\&rendertype=abstract, doi $10.1073 /$ pnas .0812581106 .

560 Munk, W.H., MacDonald, G.J.F., 2009. The Rotation of the Earth: A Geophysical Discussion. Cambridge Monographs on Mechanics, Cambridge University Press. URL: https://books.google.com/books?id=klDqPAAACAAJ.

Nettelmann, N., Püstow, R., Redmer, R., 2013. Saturn layered structure and homogeneous evolution models with different EOSs. Icarus 225, 548-557. URL: http://linkinghub.elsevier.com/retrieve/pii/S0019103513001784, doi:10.1016/j.icarus.2013.04.018, arXiv:1304.4707.

Tassoul, J.L., 2015. Theory of Rotating Stars. (PSA-1). Princeton Series in ¿ Astrophysics, Princeton University Press. URL: https://books.google. com/books?id=nnJ9BgAAQBAJ.

570 Vorontsov, S.V., Gavrilov, S.V., Zharkov, V.N., Leontev, V.V., 1984. Dynamical theory of the tides on the giant planets. Astron. Vestn. 18, 8-18.

¿Wisdom, J., 1996. Non-perturbative Hydrostatic Equilibrium URL: http:// web.mit.edu/wisdom/www/interior.pdf.

Wisdom, J., Hubbard, W.B., 2016. Differential rotation in Jupiter: A compar-

ison of methods. Icarus 267, 315-322. URL: http://dx.doi.org/10.1016/ j.icarus.2015.12.030, doi $10.1016 /$ j.icarus.2015.12.030.

Zharkov, V.N., 2004. A theory of the equilibrium figure and gravitational field of the Galilean satellite Io: The second approximation. Astron. Lett. 30, 496-

¿ 507. URL: http://link.springer.com/10.1134/1.1774402, doi 10.1134/ 1.1774402 
Zharkov, V.N., Gudkova, T.V., 2010. Models, figures and gravitational moments of Jupiter's satellite Io: Effects of the second order approximation. Planet.

口 Space Sci. 58, 1381-1390. URL: http://dx.doi.org/10.1016/j.pss.2010. 06.004 doi $10.1016 / \mathrm{j} . \mathrm{pss} .2010 .06 .004$. chart, Tucson, AZ.

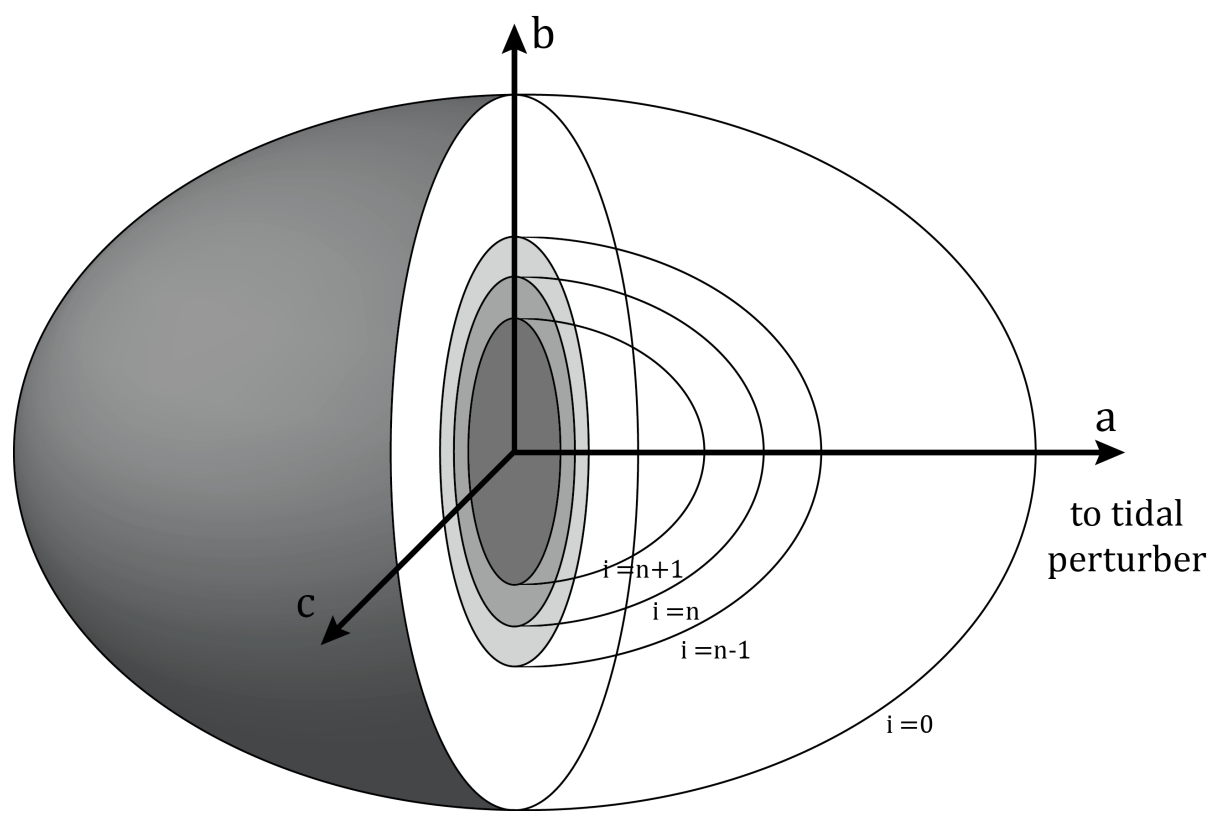

Figure 1 Conceptual diagram of a Concentric Maclaurin Spheroid (CMS) model with a tidal perturbation from a satellite. 
Table 1. Comparing two-layer models

\begin{tabular}{cccc}
\hline \hline$a_{1} / a$ & $\rho_{0} / \rho_{1}$ & $k_{2}$ CMS & $k_{2}$ Clairaut \\
\hline 0.1 & 0.5 & 1.496283 & 1.496286 \\
0.3 & 0.5 & 1.411183 & 1.411185 \\
0.5 & 0.1 & 0.465714 & 0.465716 \\
0.5 & 0.3 & 0.947967 & 0.947969 \\
0.5 & 0.5 & 1.205309 & 1.205311 \\
0.5 & 0.7 & 1.360183 & 1.360186 \\
0.5 & 0.9 & 1.461667 & 1.461669 \\
0.7 & 0.5 & 1.057405 & 1.057407 \\
0.9 & 0.5 & 1.217192 & 1.217194
\end{tabular}

Note. - Calculated $k_{2}$ for a two layer model with $q_{\mathrm{tid}}=10^{-6}, q_{\mathrm{rot}}=0$ and Tethy's $R / a$, for chosen values of ratio of radii and densities of the two layers. Results closely match the approximation using Clairaut theory in Folonier et al. (2015), Eqn. 41. 
Table 2. Saturn Model Parameters

\begin{tabular}{rrrr}
\hline \hline & Cassini & Voyager & \\
\hline$G M$ & $3.7931208 \times 10^{7} \mathrm{a}$ & $\ldots$ & $\left(\mathrm{km}^{3} / \mathrm{s}^{2}\right)$ \\
$a$ & $6.0330 \times 10^{4} \mathrm{a}$ & $\ldots$ & $(\mathrm{km})$ \\
$J_{2} \times 10^{6}$ & $16290.71^{\mathrm{a}}$ & $\ldots$ & \\
$J_{4} \times 10^{6}$ & $-935.83^{\mathrm{a}}$ & $\ldots$ & \\
$J_{6} \times 10^{6}$ & $86.14^{\mathrm{a}}$ & $\ldots$ & \\
$q_{\text {rot }}$ & $0.1516163^{\mathrm{b}}$ & $0.1553029^{\mathrm{c}}$ \\
$r_{\text {core }} / a$ & 0.2 & $\ldots$ \\
$m_{\text {core }} / M$ & 0.133146 & 0.140478 \\
\hline \hline & Tethys & Dione \\
\hline$q_{\text {tid }}$ & $-2.791103 \times 10^{-8} \mathrm{~d}$ & $-2.364582 \times 10^{-8} \mathrm{~d}$ \\
$R / a$ & $4.8892^{\mathrm{d}}$ & $6.2620^{\mathrm{d}}$ \\
\hline
\end{tabular}

References. - a. Jacobson et al. (2006), b. Giampieri et al. (2006), c. Desch and Kaiser (1981), d. Archinal et al. (2011)

Note. - Identical parameters for Saturn are used with the exception of $q_{\text {rot }}$, for which the rotation rate from both Cassini and Voyager are considered. A constant core density is fitted to match $J_{2}, J_{4}$, and $J_{6}$ for a converged figure. 
Table 3. Calculated Saturn tidal responses

\begin{tabular}{|c|c|c|c|c|}
\hline model $^{\mathrm{a}}$ & & gravitational moment & & normalized moment \\
\hline Cassini & $J_{2}$ & $1.62907100025 \times 10^{-2}$ & $J_{2} / q_{\mathrm{rot}}$ & 0.10744694879478 \\
\hline no tide & $J_{4}$ & $-9.2027941201 \times 10^{-4}$ & $J_{4} / q_{\mathrm{rot}}$ & $-0.606979160784 \times 10^{-2}$ \\
\hline$N=128$ & $J_{6}$ & $8.014294995 \times 10^{-5}$ & $J_{6} / q_{\mathrm{rot}}$ & $0.5285905549 \times 10^{-3}$ \\
\hline non-rotating & $C_{22}$ & $8.5288 \times 10^{-10}$ & $k_{2}$ & 0.36669 \\
\hline Tethys & $J_{2}$ & $1.70576 \times 10^{-9}$ & $J_{2} / q_{\mathrm{rot}}$ & . \\
\hline$N=128$ & $J_{4}$ & $-1.351 \times 10^{-11}$ & $J_{4} / q_{\mathrm{rot}}$ & $\cdots$ \\
\hline polytrope & $J_{6}$ & $2.2 \times 10^{-13}$ & $J_{6} / q_{\mathrm{rot}}$ & $\cdots$ \\
\hline Cassini & $C_{22}$ & $9.6070 \times 10^{-10}$ & $k_{2}$ & 0.41304 \\
\hline Tethys & $J_{2}$ & $1.629071017501 \times 10^{-2}$ & $J_{2} / q_{\mathrm{rot}}$ & 0.1074469499328 \\
\hline$N=128$ & $J_{4}$ & $-9.2027943932 \times 10^{-4}$ & $J_{4} / q_{\mathrm{rot}}$ & $-0.60697917880 \times 10^{-2}$ \\
\hline polytrope & $J_{6}$ & $8.01429541 \times 10^{-5}$ & $J_{6} / q_{\mathrm{rot}}$ & $0.5285905822 \times 10^{-3}$ \\
\hline Voyager & $C_{22}$ & $9.4136 \times 10^{-10}$ & $k_{2}$ & 0.40473 \\
\hline Tethys & $J_{2}$ & $1.629071048760 \times 10^{-2}$ & $J_{2} / q_{\mathrm{rot}}$ & 0.1048963407747 \\
\hline$N=128$ & $J_{4}$ & $-9.3570887868 \times 10^{-4}$ & $J_{4} / q_{\mathrm{rot}}$ & $-0.60250556585 \times 10^{-2}$ \\
\hline polytrope & $J_{6}$ & $8.30176108 \times 10^{-5}$ & $J_{6} / q_{\mathrm{rot}}$ & $0.534552720 \times 10^{-3}$ \\
\hline Cassini & $C_{22}$ & $8.1325 \times 10^{-10}$ & $k_{2}$ & 0.41272 \\
\hline Dione & $J_{2}$ & $1.629071019035 \times 10^{-2}$ & $J_{2} / q_{\mathrm{rot}}$ & 0.1074469500340 \\
\hline$N=128$ & $J_{4}$ & $-9.2027943688 \times 10^{-4}$ & $J_{4} / q_{\mathrm{rot}}$ & $-0.60697917719 \times 10^{-2}$ \\
\hline polytrope & $J_{6}$ & $8.01429534 \times 10^{-5}$ & $J_{6} / q_{\mathrm{rot}}$ & $0.528590578 \times 10^{-3}$ \\
\hline Cassini & $C_{22}$ & $9.6219 \times 10^{-10}$ & $k_{2}$ & 0.41368 \\
\hline Tethys & $J_{2}$ & $1.629071019560 \times 10^{-2}$ & $J_{2} / q_{\mathrm{rot}}$ & 0.1074469500686 \\
\hline \multirow[t]{2}{*}{$N=4$} & $J_{4}$ & $-9.3583002600 \times 10^{-4}$ & $J_{4} / q_{\mathrm{rot}}$ & $-0.61723571821 \times 10^{-2}$ \\
\hline & $J_{6}$ & $8.61400043 \times 10^{-5}$ & $J_{6} / q_{\mathrm{rot}}$ & $0.568144705 \times 10^{-3}$ \\
\hline Cassini & $C_{22}$ & $9.6235 \times 10^{-10}$ & $k_{2}$ & 0.41375 \\
\hline Tethys & $J_{2}$ & $1.629070920013 \times 10^{-2}$ & $J_{2} / q_{\mathrm{rot}}$ & 0.1074469435029 \\
\hline$N=511$ & $J_{4}$ & $-9.3582993628 \times 10^{-4}$ & $J_{4} / q_{\mathrm{rot}}$ & $-0.61723565903 \times 10^{-2}$ \\
\hline DFT-MD & $J_{6}$ & $8.09366588 \times 10^{-5}$ & $J_{6} / q_{\mathrm{rot}}$ & $0.533825538 \times 10^{-3}$ \\
\hline Voyager & $C_{22}$ & $9.4185 \times 10^{-10}$ & $k_{2}$ & 0.40495 \\
\hline Tethys & $J_{2}$ & $1.629070988378 \times 10^{-2}$ & $J_{2} / q_{\mathrm{rot}}$ & 0.104896336887 \\
\hline$N=511$ & $J_{4}$ & $-9.3583000384 \times 10^{-4}$ & $J_{4} / q_{\mathrm{rot}}$ & $-0.60258355868 \times 10^{-2}$ \\
\hline DFT-MD & $J_{6}$ & $8.16661484 \times 10^{-5}$ & $J_{6} / q_{\mathrm{rot}}$ & $0.525850615 \times 10^{-3}$ \\
\hline
\end{tabular}

${ }^{\mathrm{a}}$ Models are denoted by: rotation rate from Cassini or Voyager, satellite parameters for Tethys or Dione, number of layers $N$, and the equation of state used. DFT-MD refers to the H-He equation of state from Hubbard and Militzer 2016. 


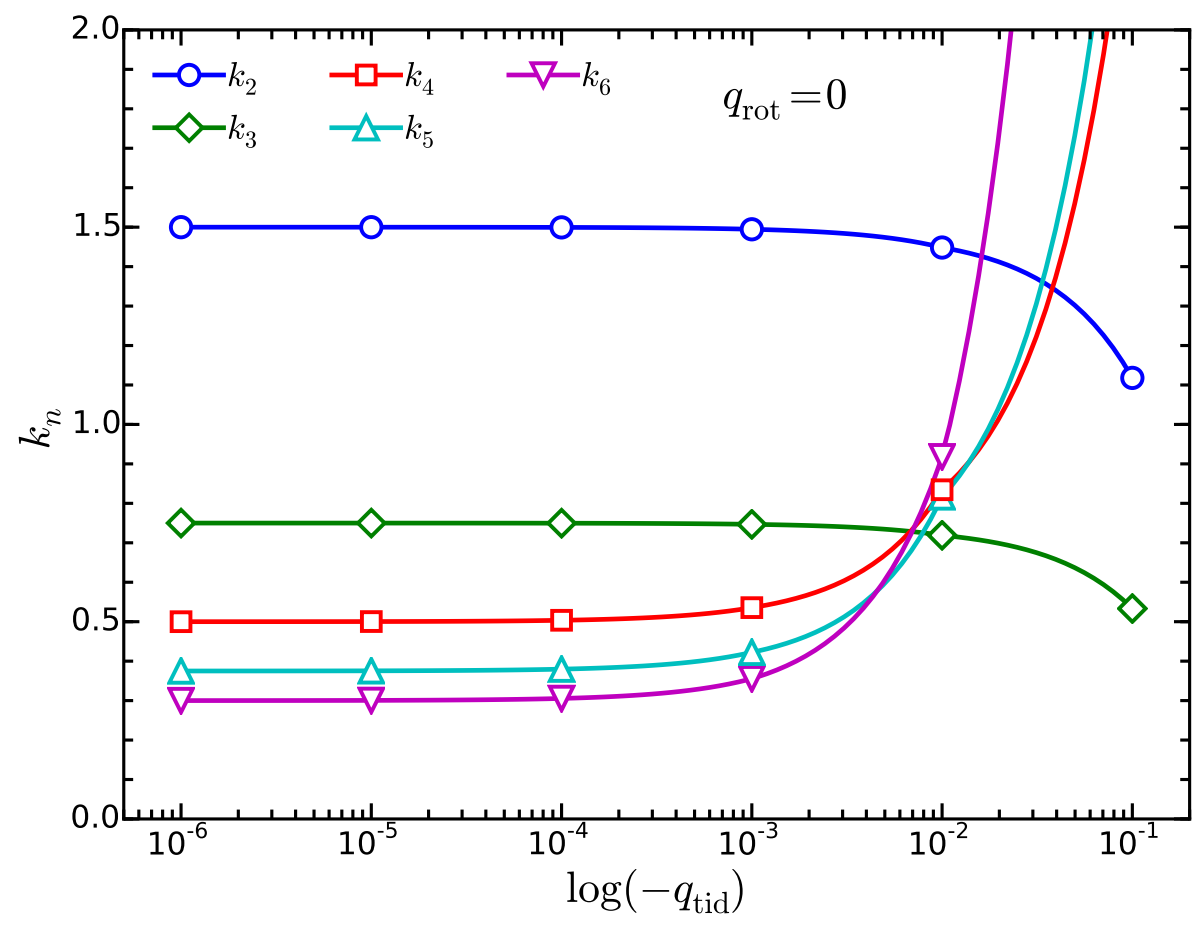

Figure 2 The effect of tidal perturbation strength on the tidal love numbers of a non-rotating constant density (Jeans) spheroid up to order 6 . The love numbers $k_{n}$ are degenerate with respect to $m$. The orbital radius is taken to be that of Tethys. 


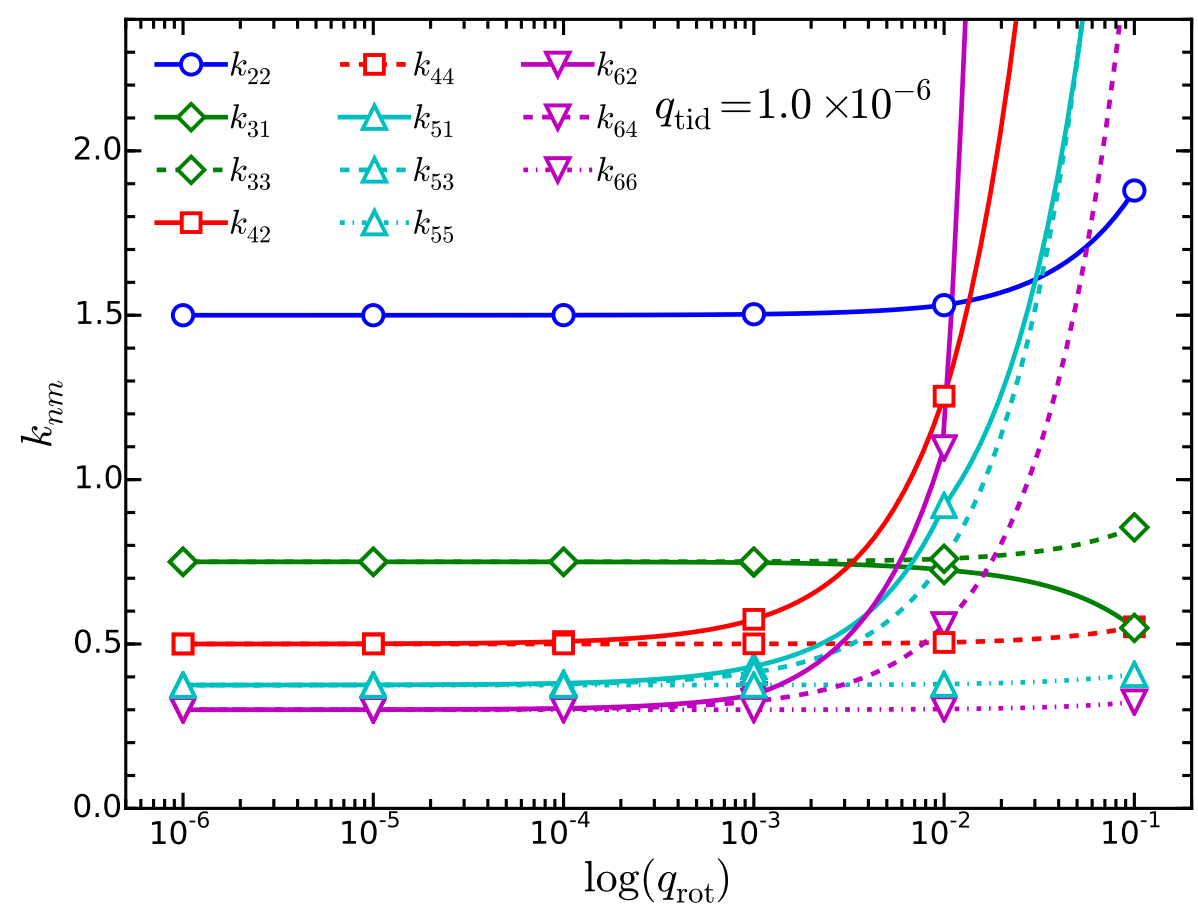

Figure 3 The effect of rotation rate on the tidal love numbers of a constant density (Roche) spheroid up to order 6 . The $k_{n m}$ for a given $n$ are found to split at high rotation rates. $q_{\text {tid }}$ is kept constant at $1.0 \times 10^{-6}$, and the orbital radius is taken to be that of Tethys. 


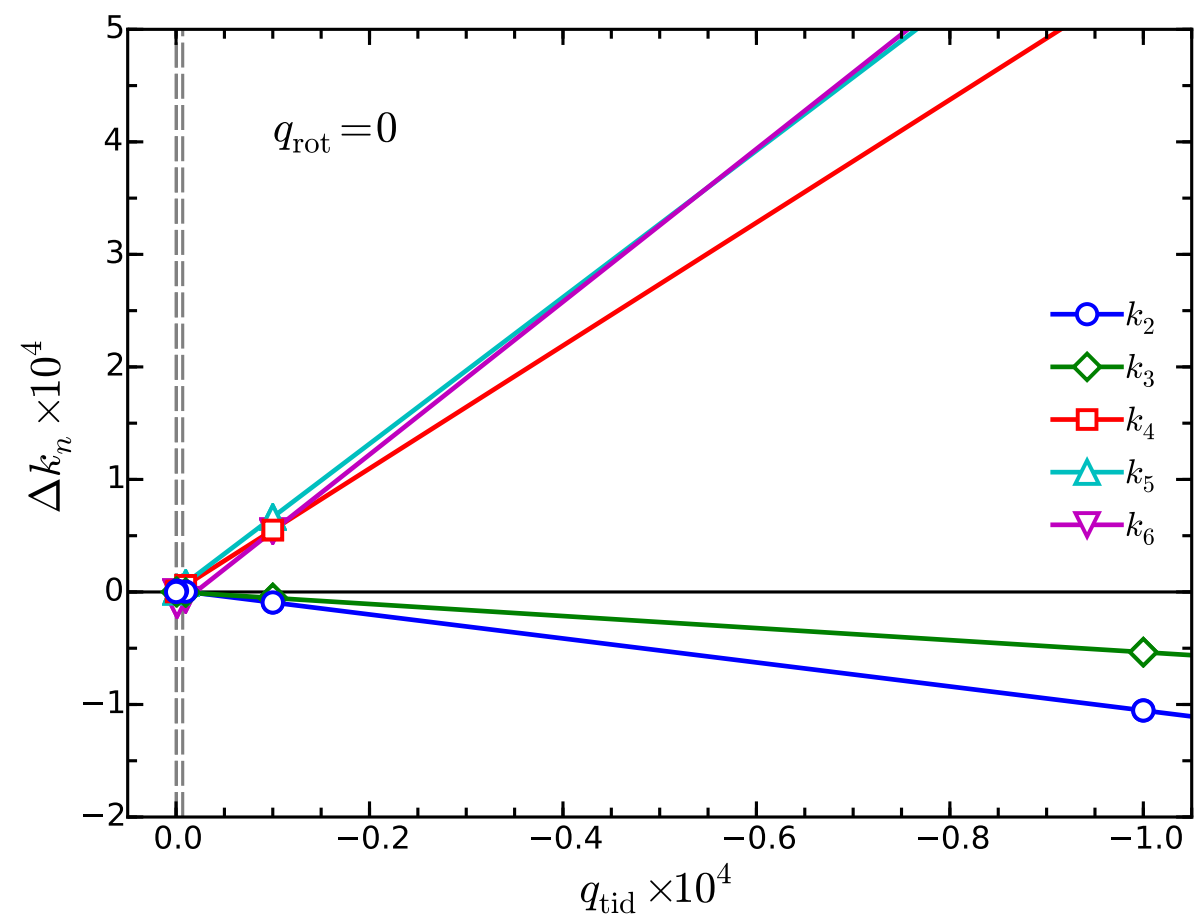

Figure 4 The effect of tidal perturbation strength on the tidal love numbers of a non-rotating planet with an $n=1$ polytrope equation of state, up to order 6. $\Delta k_{n}$ is the shift in love number $k_{n}$ from the limit of low $q$ tid. The love numbers $k_{n}$ are degenerate with respect to $m$. The orbital radius is taken to be that of Tethys. The vertical, dashed gray lines show $q_{\text {tid }}$ for Tethys-Saturn and Io-Jupiter. 

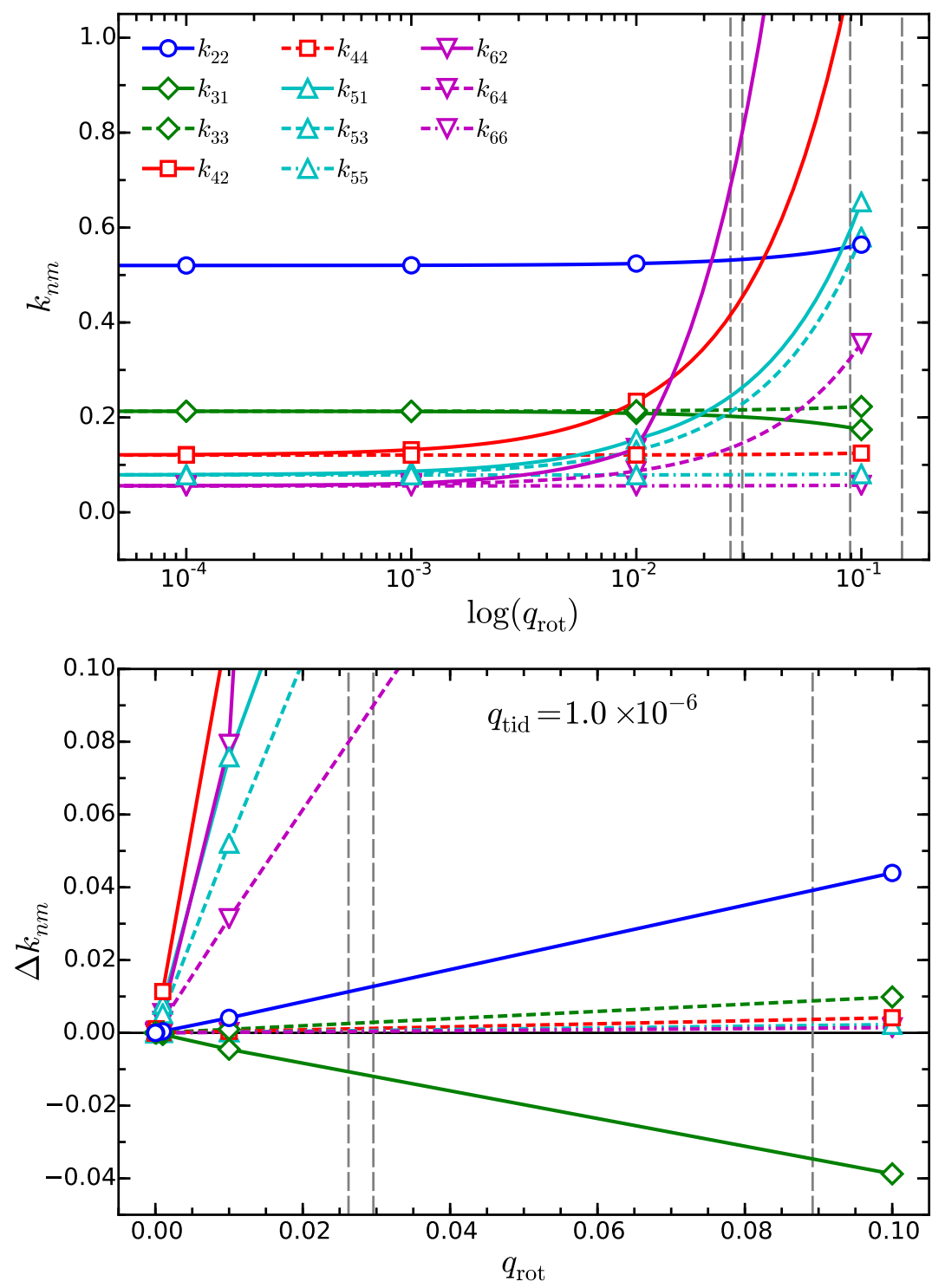

Figure 5 Top: The effect of rotation rate on the tidal love numbers of a planet with an $n=1$ polytrope equation of state, up to order 6 . The $k_{n m}$ for a given $n$ are found to split at high rotation rates. $q_{\text {tid }}$ is kept constant at $1.0 \times 10^{-6}$, and the orbital radius is taken to be that of Tethys. The vertical, dashed gray lines show $q_{\text {rot }}$ for Neptune, Uranus, Jupiter and Saturn. Bottom: Shift in $k_{n m}$ as a function of $q_{\text {rot }}$ on a linear scale. 


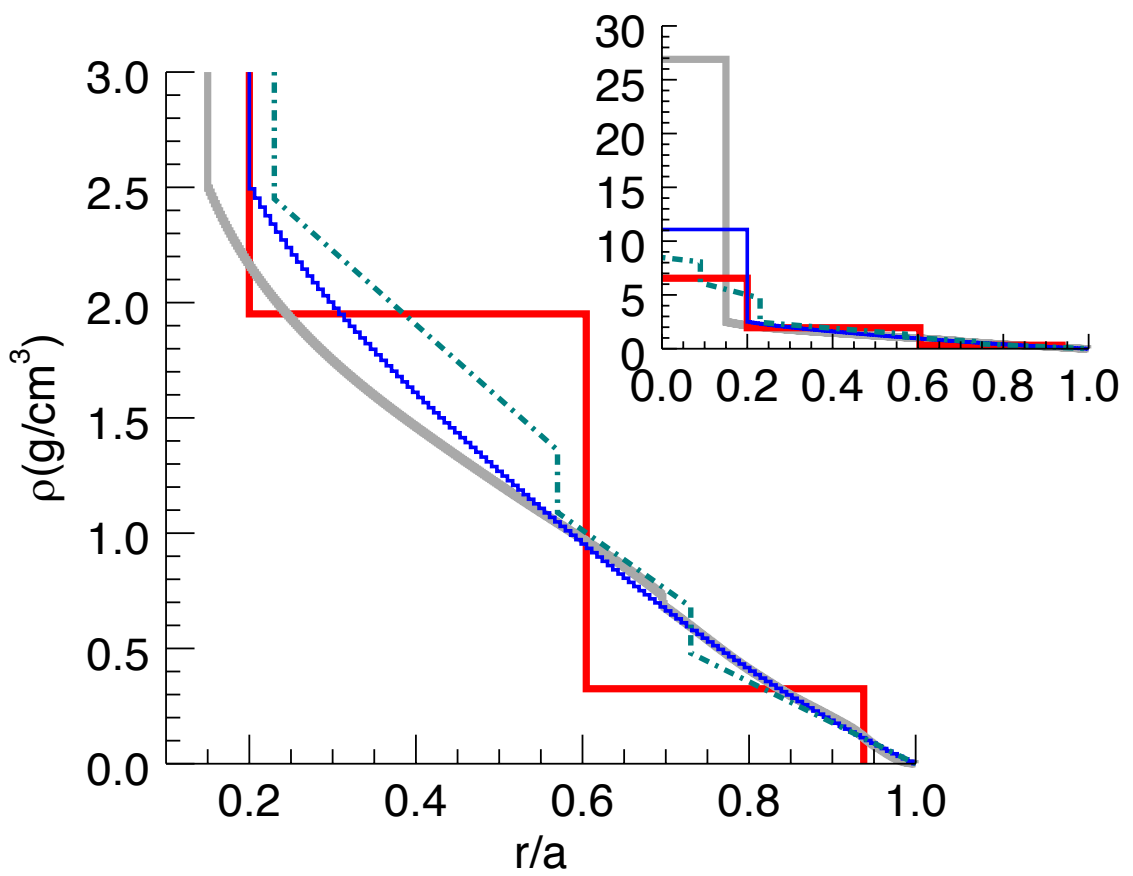

Figure 6 Density structure of simple Saturn models, all fitted to Saturn's observed $J_{2}$ (Jacobson et al., 2006). The blue curve shows an $N=128$ model with a constant-density core within $r=0.2 a$ and a polytropic outer envelope. The red curve shows an $N=4$ model with the same core radius and two additional spheroids, adjusted to fit both $J_{2}$ and $J_{4}$. For comparison, the dash-dot curve (teal) shows Saturn model MS24 of Gudkova and Zharkov (1999). The grey solid curve shows an unpublished Saturn model based on the density-functional theory, molecular-dynamics (DFT-MD) equation of state for hydrogen-helium, as used in the Jupiter model of Hubbard and Militzer (2016). 


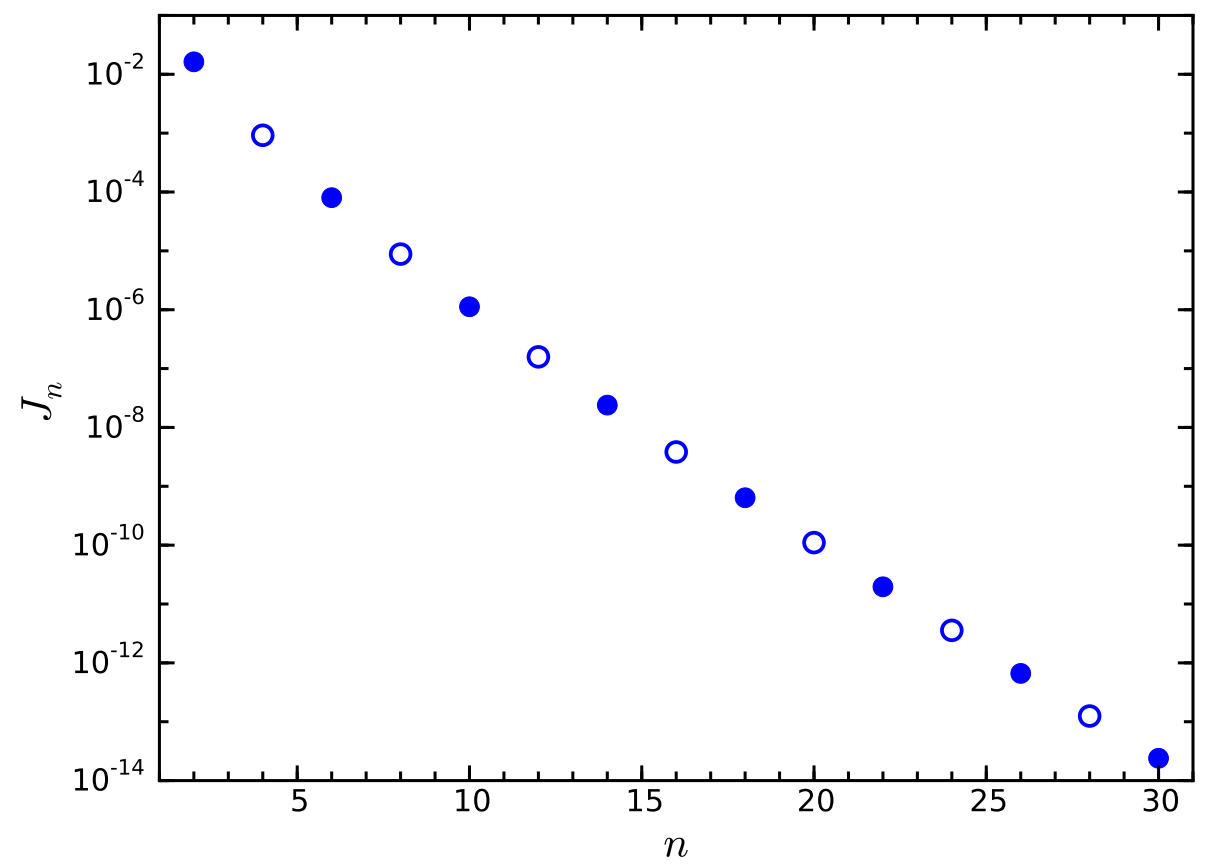

Figure 7 The zonal harmonics $J_{n}$ for the Cassini Saturn model. Positive values are shown as filled and negative as empty. 


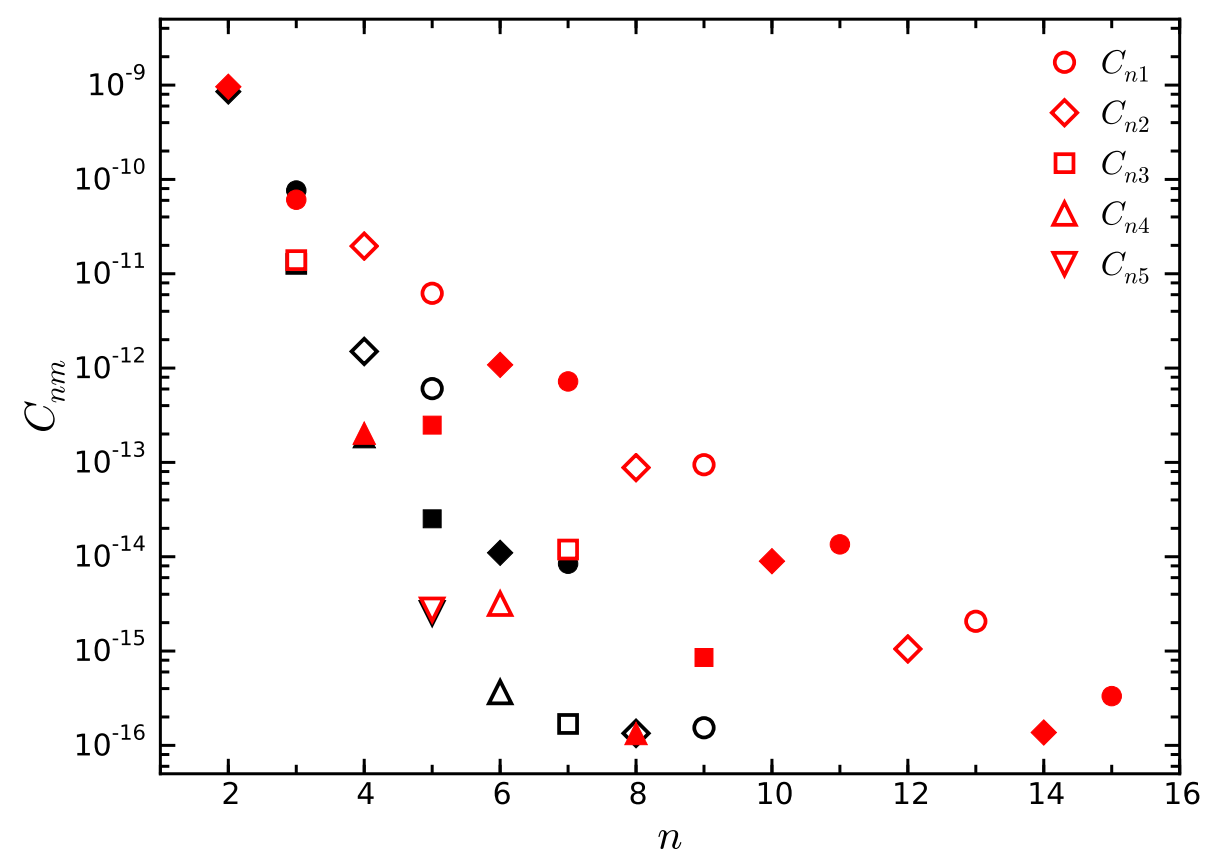

Figure 8 In red, the tesseral harmonics $C_{n m}$ for the Cassini Saturn model. In black, $C_{n m}$ for the same density profile and same value of $q_{\mathrm{tid}}$, but with $q_{\mathrm{rot}}=0$. Positive values are shown as filled and negative as empty. 